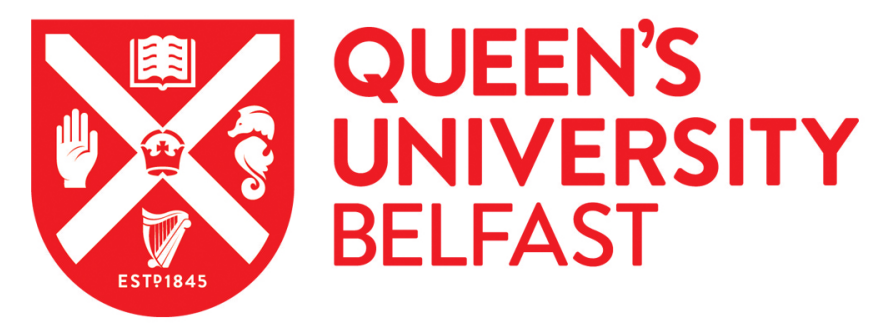

\title{
Long-acting implantable devices for the prevention and personalised treatment of infectious, inflammatory and chronic diseases
}

Corduas, F., Mancuso, E., \& Lamprou, D. (Accepted/In press). Long-acting implantable devices for the prevention and personalised treatment of infectious, inflammatory and chronic diseases. Journal of Drug Delivery Science and Technology.

Published in:

Journal of Drug Delivery Science and Technology

Document Version:

Peer reviewed version

Queen's University Belfast - Research Portal:

Link to publication record in Queen's University Belfast Research Portal

Publisher rights

Copyright 2020 Elsevier.

This manuscript is distributed under a Creative Commons Attribution-NonCommercial-NoDerivs License

(https://creativecommons.org/licenses/by-nc-nd/4.0/), which permits distribution and reproduction for non-commercial purposes, provided the author and source are cited.

\section{General rights}

Copyright for the publications made accessible via the Queen's University Belfast Research Portal is retained by the author(s) and / or other copyright owners and it is a condition of accessing these publications that users recognise and abide by the legal requirements associated with these rights.

Take down policy

The Research Portal is Queen's institutional repository that provides access to Queen's research output. Every effort has been made to ensure that content in the Research Portal does not infringe any person's rights, or applicable UK laws. If you discover content in the Research Portal that you believe breaches copyright or violates any law, please contact openaccess@qub.ac.uk. 


\section{Long-acting implantable devices for the prevention and personalised treatment of infectious, inflammatory and chronic diseases}

Francesca Corduas ${ }^{1,2}$, Elena Mancuso ${ }^{1}$, Dimitrios A. Lamprou ${ }^{2, *}$

${ }^{1}$ Nanotechnology and Integrated Bio-Engineering Centre (NIBEC), Ulster University, Jordanstown Campus - Newtownabbey BT37 0QB, UK.

${ }^{2}$ School of Pharmacy, Queen's University Belfast, Belfast BT9 7BL, UK

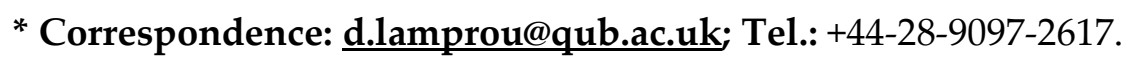

Abstract: Traditional drug administration methods, including injection, oral and nasal route, have been extensively adopted over the years due to their low-cost and/or easy ways to deliver drugs. However, most of the times, they are not effective and significant drawbacks still compromise their application. In order to overcome the shortcoming associated with traditional administration routes, many researchers have been focusing their attention on long-acting biomedical implants, which have gained great interest within the scientific community in the last decade. These are usually intended to support the functions of a specific organ by slowly releasing a medication or simply monitor body functions. One of the most promising fields of application of such devices is the treatment of chronic diseases, long-lasting infections (e.g. human immunodeficiency virus (HIV)) and chronic inflammation in wound healing (implants in form of dressings). In this regard, the availability of new manufacturing approaches and novel biocompatible materials have positively contributed to design innovative personalised and targeted drug delivery systems (DDSs). Among all the benefits associated with the use of new DDSs, an improvement in patient's compliance (or adherence) to the medication is the most significant. This review aims to provide an overview of the recently developed technologies for the prediction, prevention, and personalised treatment (3Ps) of human 
immunodeficiency virus (HIV), cardiovascular diseases (CVDs), diabetes and chronic wounds, focusing on long-acting biomedical implants and their promise for future application.

Keywords: 3D Printing; chronic diseases; drug delivery; electrospinning; implantable devices; infections; inflammatory; long acting; microfluidics; personalized therapy.

\section{General Introduction}

The technological development in the biomedical field has made possible the production of a wide range of new bio-devices. Especially, the availability of new manufacturing methods and biocompatible materials has allowed to design innovative personalised and targeted drug delivery systems (DDSs) [1]. The DDSs can be made of both degradable and non-degradable materials, varying the mechanism of drug release according to this specific feature. In degradable systems, the medication delivery is mainly due to the erosion (bulk and surface) of the device, swelling of the material and diffusion, while in non-degradable systems; release is only due to diffusion. Moreover, other external parameters (e.g. $\mathrm{pH}$, temperature, drug physicochemical properties) can influence the time and modality by which drugs are delivered and must be considered during the design process of the device [2]. This is an important step forward in research, since DDSs permit to tightly control the drug release rate, as well as its specific delivered amount and the target site [3]. The older routes of administration, such as injection and oral, present some disadvantages linked to the lack of control over the timing of release, as well as a reduced therapeutics' bioavailability and stability and impossibility of removal in case of adverse reaction [4].

Among all the benefits associated with the use of new DDSs, an improvement in patient's compliance (or adherence) to the medication is one of the most significative. 
Using the latest platforms for therapeutics delivery could be a powerful choice to improve this aspect, making therapies personalised and more patient-friendly $[2,4]$.

Many researchers have now focused their attention on long-acting biomedical implants, which intend or to replace the functions of a specific organ by slowly releasing the medication, or to replace the organ itself, or simply monitor body functions [5]. One of the most promising fields of application of such devices is the treatment of chronic diseases [6], long-lasting infections (e.g. human immunodeficiency virus (HIV)) [7] and chronic inflammation in wound healing (implants in form of dressings) [8]. Very often, to treat these pathologies, daily drug administration is required, and in this view biomedical implants perfectly fit [7, 9].

To date, a wide range of new manufacturing methods are available, starting from additive manufacturing (e.g. 3D printing), microfluidics, and electro-hydrodynamic processes (e.g. electrospinning), arriving to microelectromechanical systems (MEMS). These technologies are very up-and-coming both for treatment and monitoring, allowing not only to produce devices with better performances, but also the creation of smart DDSs that can be successfully employed in the field of personalized medicine, tailoring the therapy from patient to patient $[3,10,11]$.

In this review will be given an overview of the recently developed technologies for the prediction, prevention, and personalised treatment of human immunodeficiency virus (HIV), cardiovascular diseases (CVDs), diabetes and chronic wounds, focusing on long-acting biomedical implants. The investigated devices could be profitably personalized according to the patient's medical history and needs, aiming to administer drugs in an even more friendly and comfortable way. 


\section{Human immunodeficiency virus (HIV)}

\subsection{HIV Pathophysiology and epidemiology}

Human Immunodeficiency Virus (HIV) is a lentivirus (subgroup of retrovirus) [12] that started to spread among the worldwide population in 1980s [13]. The HIV morphology is characterised by the presence of a lipid bilayer membrane in which three trimer of glycoprotein 41 (Gp41) and glycoprotein 120 (Gp120) are embedded, constituting the viral envelope that the virus used to bind to some specific receptors of the host cell and start its viral activity [12]. Particularly, HIV prefers to bind to CD4 and CCR5 receptor $[12,14]$ that mainly presents on T lymphocytes surface [14].

The virus can enter the cell by membrane fusion or endocytosis, releasing its viral RNA in the cytoplasm and, subsequently, by fusing it with non-viral DNA. [12] This process produces infected $\mathrm{T}$ cells that die due to apoptosis or pyroptosis (high inflammatory programmed cell death), causing a significant decrease in their number [15]. When the CD4 T lymphocytes concentration falls below a certain threshold (200 cells $\mu \mathrm{L}^{-1}$ ), then the immune system is no more able to counteract the infection leading to the progression of AIDS [16].

In the last 40 years, substantial progress has been made for the treatment of this condition [13]. Therapeutic approaches, such as Pre-exposure Prophylaxis (PrEP) and Anti-retroviral Therapy (ART), have been approved [13, 17], allowing the infected people to live a "normal" life [18]. ART and PrEP consist in the administrations of antiretroviral drugs (ARV) but PrEP is used to block the acquisition of the virus before the exposure, while ART is used in order to treat AIDS [19] (Fig.1).

The development of PrEP, which is a prevention strategy for uninfected individuals at risk, allowed to reduce the risk of contraction by $85 \%$, while ART resulted in the suppression of the virus in $80 \%$ of the infected patients [21]. 
Aiming to reduce the global cases of HIV infection and in order to put the maximum effort in research, in 2014 the Joint United Nation Programme on HIV and AIDS set the 90-90-90 targets. The purpose was to make aware of their status on $90 \%$ of the population, to start ART on the $90 \%$ of the diagnosed patients and to virally suppress the virus in the $90 \%$ of the cases by the year 2020 [17]. Unfortunately, 1.8 million people contract the virus every year [22] and to date, approximately 37.9 million people live with HIV [23].

One of the cause for such numbers is due to the poor adherence of the majority of patients to the daily administration of ARV [24]. Even short periods of non-adherence can be extremely detrimental and drastically reduce the health benefits of the therapy [25]. Thus nowadays, the main goal of research is to develop long-acting injectable drugs and long-acting implantable devices that allow for infrequent dosing (non-daily administration) $[7,25]$.

Long-acting injectable drugs are commonly administrated via intramuscular (IM) administration [26]. Their main advantages are linked to the possibility of avoiding the gastrointestinal (GI) tract and all the adverse events that are related to this route of administration such as drug-drug interactions and first pass metabolism [26]. However, this kind of route also shows some drawbacks, mainly due to the impossibility of removing the drugs from the circulation in case of adverse reaction [24]. However, implantable devices have shown some improvements: can be removed in case of adverse reaction, provide a predictable release profile, can be biodegradable avoiding removal and do not produce pharmacokinetic tails [7]. Due to many benefits of using implantable devices, research is now focusing on the development of new long-acting implantable devices, especially for prevention applications [27].

\subsection{Long acting drugs for HIV}

One of the main strategies that can be employed to manage the poor adherence of the PrEP therapy from the patients is by using long acting drugs. In order to be suitable 
for infrequent treatments, these drugs must have some features, such as be potent and insoluble allowing infrequent administration (due to their poor water solubility) and also a dose reduction [28]. These are key aspects especially with regards to implants, due to the limited capacity of the implant's reservoir [7]. Tenofovir (TVF) and Cabotegravir $(\mathrm{CAB})$ are some examples very often used in combination with implants.

TVF is a Nucleoside Reverse Transcriptase Inhibitor (NRTI) with a half-life of 60$100 \mathrm{~h}$, and it is used due to its safety and tolerability [21]. It can be administered not only in form of TVF, but also in form of other two prodrugs such as tenofovir disoproxil fumarate (TDF) and tenofovir alafenamide fumarate (TAF) [29]. Particularly, with regards to TAF, it has been proven that it is 10 times stronger than TDF [7, 21], less toxic to kidneys and bones [18, 30] and also, it shows activity against Hepatitis B virus [7].

$\mathrm{CAB}$ is a HIV integrase inhibitor [21] with a strong antiviral activity [31] and currently it is in phase III clinical trials [29]. It is suitable both for oral and long-acting injectable administration [21, 30, 31], with a half-life of 31.5h [21]. It has been proven that $\mathrm{CAB}$ is able to reach an optimal plasma concentration, in both men and women, when administered every 8 weeks [31]. Also, it has very low interaction with other drugs [31], it is well tolerated and its use is related with mild adverse effects at the injection site [21, 30].

ART is not able to completely defeat the viral reservoir, and this is why new drugs are still under investigation [32]. One example is broadly neutralizing antibodies (bNABs) [32]. These are commonly administrated in form of crystals through injections [33], but also have been employed in the design process of new types of implantable DDSs, such as intravaginal rings (IVR) [34]. bNABs isolated from the infected patients [35] show a very high level of potency and wide neutralization breadth against a vast number (almost 80\%) [30] of HIV strains [36]. They have an half-life of 2-3 weeks [37] and their outcomes can be improved through combination of different types [30,37] or through modulation of their fragment crystallisable $\left(F_{c}\right)$ region $[32,33]$. 


\subsection{Implants for the delivery of HIV drug molecules}

Implants presented in this section, can be divided in polymer-based (e.g. intravaginal and subcutaneous implants) and nanofluidic-based (e.g. refillable devices).

Intravaginal rings (IVR) are included in the class of topical drug delivery implants and are designed in order to be user-friendly and continues monitoring of the disease/therapy [38]. A Pod-intravaginal ring able to deliver TDF in combination with maraviroc (MVC), an inhibitor/antagonist of the receptor CCR5, was developed by Moss et al. [39]. The Pod-intravaginal ring was made by 10 pods, 6 containing TDF and 4 containing MVC, that acted as independent reservoirs [39]. A pharmacokinetic analysis was performed in an ovine model and the results showed that the device was able to deliver TDF and MVC at a constant rate for almost 28 days, by keeping for the whole duration of the release a significative concentration of both substances in the sheep cervicovaginal fluid. Also, it was found that the steady state concentration was reached in almost $24 \mathrm{~h} \mathrm{[39].}$

In addition to this, Ugaonkar et al. produced a core-matrix intravaginal ring by combined four pharmaceutical compounds (MIV-150 and zinc acetate against HIV-1 and HIV-2, carrageenan against human papilloma virus HPV, and levonorgestrel as contraceptive). Their aim was to obtain an implantable device able to protect the patient against two types of HIV virus (HIV-1 and HIV-2), HPV and pregnancy. The drug molecules continuously diffused from the device for almost 94 days in vitro and 28 days in vivo. Moreover, drug levels were enough to counteract the effects of the viruses and unintended pregnancy (Fig.2) [40].

Keeping in mind all the possible strategies that can be exploited to face HIV infections, Zhao et al. produced a bNABs-loaded IVR capable of releasing nicotinemanufactured VRC01-N antibodies in vivo. The device contained several pods coated with polylactide acid (PLA), which acted like rate controlling membrane, and then 
embedded in silicon ring scaffold. Each pod was provided with a channel to allow diffusion from the reservoir. Drug concentration levels were satisfactory (steady state levels in vaginal fluid: $10^{2}-10^{3} \mu \mathrm{g} \mathrm{g}^{-1}$ ) for up to 21 days. As each pod act like a distinct reservoir with independent release characteristics, this device can be also used to deliver multiple combination of mAbs [34].

IVRs are the gold standard in the treatment of HIV infection in the female population, but patient's adherence to their administration is still a concern; moreover, manufacturing of IVRs is usually carried on at high temperatures, making the incorporation of therapeutics difficult. Recently, elecrospun fibrous scaffolds started to be used in HIV treatment [41]. Electrospinning is a manufacturing technique that allow to produce micro/nanometric fibres by exploiting electric field. It gained attention because electrospun devices have a great extracellular matrix (ECM) mimicking ability, promoting cells attachment, migration and proliferation. Also, it allows to simply include drugs in the formulation, creating drug-loaded devices that can be employed in a variety of biomedical applications [42]. A poly (lacti-co-glycolic acid) (PLGA) electrospun matrix was produced by Grooms et al. in order to provide a barrier and to inactivate the virus prior to cell entrance. The device was functionalized by surface modification of the fibres integrating the antiviral protein griffithsin (GRFT). The same group demonstrated that GRFT has a great potential not only against HIV, but also against herpes simplex virus (HSV), making their device multipurpose. In addition, topically applied GRFT may be customized to bind a variety of pathogen target (e.g. Gp120) and release multiple antiviral agents (e.g. genetic agents and antivirals). GRFT was tested at different concentrations $(0.00005,0.0005,0.005,0.05,0.5,5 \mathrm{nmol} / \mathrm{mg})$ and in vitro experiments were performed to assess its potency, also cytotoxicity assays against cervical epithelial cells were carried on in order to test their viability. Antiviral activity was reached with 0.05 , 0.5 and $5 \mathrm{nmol} / \mathrm{mg}$, additionally these concentrations were not toxic at all for the chosen cell lines (VK2, Ect1, End1) [43]. 
Another approach to PrEP includes the possibility to insert subcutaneous implants. Johnson et al. developed a TAF-eluting polycaprolactone (PCL) tube, fabricated via single-screw hot-melt extrusion (HME) process. The biodegradable device showed a zero-order release kinetics. The delivery of the drugs was mainly controlled by the morpho-chemical properties of the device, such as surface area, wall thickness and PCL crystallinity, thus allowing the fine tuning of the implant parameters in order to personalize it, in terms of dosing and duration, according to some specific clinical and socio-behavioural conditions (Fig.3) [44].

Polymer-based devices can be made of biodegradable or non-biodegradable materials, moreover they can require removal after drug exhaustion [25], which is the main reason why refillable nanofluidic-based devices are gaining attention [45]. Micro/nanofluidic platforms are characterized by micro/nano-scale channels and they were recently employed in drug delivery applications thanks to their advantages, which include low costs, portability, reproducibility, the possibility to tightly control the device microenvironment and drug release [3,46]. A refillable nanofluidic implant was developed by Chua et al. with experiments carried on two titanium devices constituted by two refillable reservoirs containing TAF and emtricitabine (FTC), which can release drugs through silicon membranes (circular and rectangular shaped). To perform in vivo studies, the implants were inserted in the dorsal scapular region with the membrane side facing the dermis, in such a way to make the device recognizable and palpable. The implants had also the ability to be detected through ultrasound imaging. From the results was possible to ascertain the ability of the device to successfully deliver TAF and several problems in the delivery of FTC, due to its poor half-life [45] (Fig.4).

Pons-Faudoa et al. created a nanofluidic device for the delivery of two different formulations of Cabotegravir, $\mathrm{CAB}$ and 2-Hydroxypropyl- $\beta$-cyclodextrin $\mathrm{CAB}(\beta \mathrm{CAB})$ with improved solubility. Two materials were utilized to produce the implants: polyether ether ketone (PEEK) for $\beta C A B$ and a smaller titanium reservoir for $\mathrm{CAB}$. Release was 
made possible through microfabricated silicon membrane equipped with $13 \mathrm{~nm}$ size channels densely organized in squared arrays. In vivo testes were carried out on rats, and results exhibited 3 months sustained release and significant plasma concentration of both $\mathrm{CAB}$ and $\beta C A B$, with higher values for the latter [22].

\section{Cardiovascular Diseases (CVDs)}

People affected by HIV have a great risk to develop cardiovascular diseases (CVDs) compare with non-infected people. This is primarily due to development of ART and the subsequent aging of the HIV-infected population [47]. In the following section this connection will be further investigated. Additionally, new technologies employed in the management of CVDs (with particular regard to coronary heart disease (CHD) will be shown.

\subsection{CVD Pathophysiology and epidemiology}

CVD is a class of diseases that involves blood vessels and/or heart [48]. The most common types include aneurysm, atherosclerosis, ischemic heart attack, myocardial infraction and sudden cardiac death [49]. The major risk factors associated with these pathological conditions are mainly in the aging process that leads to an increase in the plasma level of cholesterol and hypertension. However, they are associated with some external habits, like smoking or physical inactivity, or even associated pathologies, such as diabetes and obesity [50].

CVDs are the principal cause of death globally, with a number of people dying annually from CVDs than from any other cause: in 2016 only, about 17.9 million people died from CVDs [51]. Recently, has been also discovered that CVDs (especially hypertension) are among the most dangerous comorbidities associated with death by SARS-Cov-2 infection [52]. Indeed, this viral infection is associated with vascular 
inflammation, myocarditis, and cardiac arrythmias, which can subsequently aggravate pre-existing pathological conditions [53].

Among CVDs, CHD is gaining attention because it is responsible for the $30 \%$ of all global deaths and more specifically, for the $19 \%$ and $20 \%$ of deaths among the male and female worldwide population, respectively [54].

CHD directly derives from atherosclerosis and is characterised by a progressive narrowing of the coronary vessel's walls, thus affecting the oxygen supply to the heart and leading to heart failure, arrhythmias and acute coronary syndromes (ACSs), such as myocardial infractions and unstable angina [55]. Particularly, ACS can be caused by the sudden erosion or disruption of an atherosclerotic plaque, which is followed, most of the times, by thrombus formation and reduction in blood flow [56]. In the presence of a complete occlusion, patient generally present an ST-segment elevation in the electrocardiographic path, leading to myocardial infraction (STEMI), while in the case of partial occlusion, there could be a lack of ST-segment elevation leading to unstable angina (NSTEMI or UA) [57].

CHD is also one of the main cause of death among HIV-infected patients, who have $50 \%$ more chances to experience a myocardial infraction compared with uninfected people [58]. ART have significantly prolonged HIV patients' life, so they will not only suffer by HIV symptoms, but also the aging process and all the related "side effects" [59]. Moreover, ART is not able to suppress the virus so individuals must continue to take these drugs during their whole lifespan, contributing to the creation of a state of chronic inflammation and immune activation and paving the way for the arising of atherosclerotic events [60]. Atherosclerotic plaque formation results from endothelial injury and low-density lipoprotein accumulation within the arterial wall and their oxidation [61]. The injury triggers the activation of immune cells, such as monocytes, that, once migrated in the sub-endothelial space, differentiate into macrophages, encapsulate the oxidized lipoprotein and contribute to further lipid accumulation and formation of 
foam cells [61, 62]. Furthermore, in response to this inflammatory process, smooth muscle cells migrate into the luminal space of the vessel and convert fatty deposit into a fibrous capsule [62]. Lymphocytes and especially T-cells are also involved in this process [61], thus making clear the implications of HIV virus. In fact, it has been proven that the level of CD4 T-cells is strongly correlated to the number of vulnerable plaques in HIV patients [63]. Up to now, the most diffused treatment for CHD relies on the use of stents [64].

\subsection{CVD Current approaches}

The use of cardiovascular stents involves the practice of an invasive surgical procedure called percutaneous coronary intervention (PCI) [65]. This is followed by the application of dual antiplatelet therapy (DAPT), with the aim to reduce any further ischemic event [66]. Currently, different types of stents include metallic, drug eluting, biodegradable, and electronic.

Bare metal stents (BMSs) were introduced right after angioplasty balloons (BAs) in order to prevent vessel's elastic recoil [68], a phenomenon characterised by the loss of the luminal areas following overstretch of the vessel wall [69]. They can be balloonexpandable or self-expandable [65]. Even if they showed some remarkable improvements with respect to the older technologies, many disadvantages including stent thrombosis and restenosis are still associated with their use [65]. The former condition involves the development of a thrombus that can result in vessel closure [70], the latter is linked to a reduction of the vessel's lumen due to an excessive growth of tissue in the luminal side (neointimal hyperplasia) as well as to a new atherosclerotic process (neo-atherosclerosis) [69].

To overcome these issues, BMSs with thinner struts, high radiopacity, and made of new materials (cobalt-chromium and platinum-chromium alloys instead of stainless steel) were produced, unfortunately these changes did not bring any improvement [71]. 
Following on the still open challenges, drug eluting stents (DESs) were introduced, finally reducing the frequency of these events [68].

In DESs, drug molecules (e.g. Paclitaxel, Sirolimus and Sirolimus-derived) are encapsulated in the metal backbone of the stent by linking them on the implant's surface, by using carrier, or by embedding the device in a durable polymeric materials, that acted as reservoir [65]. The used drugs can be classified as immunosuppressive, antiproliferative, anti-inflammatory, antithrombotic and pro-healing agents $[72,65,73]$.

Paclitaxel is an anti-proliferative drug already employed in the treatment of some types of cancer (e.g. lung, breast and ovarian cancer) and used in this case to prevent instent restenosis [74]. Particularly, it inhibits muscle cells proliferation [73]. Sirolimus, is an antibiotic with anti-proliferative and anti-inflammatory properties, very similar to Paclitaxel and both inhibit smooth muscle cell proliferation, but with very different mechanism of action as they block the mitosis at two different stages [65, 72].

Despite all the advantages in respect to classical BMSs, the use of DESs is still subjected to problems particularly late-stent thrombosis, as prolonged DAPT has been required [66], but also inflammatory reaction against the polymeric coating [76]. These evidences have clearly showed that these technologies still present many limitations, thus calling for new approaches and devices to be used.

\subsection{New technologies for CVD}

Aiming to solve the aforementioned problems, one solution can be the employment of absorbable materials, both as coating in DES and as stent backbone [77]. It has been proven that leaving the stent in the vessel for over 9 months is not beneficial in terms of vessel re-epithelialisation and inflammatory response [65]. Nogic et al. demonstrated that biodegradable DESs, for instance PLLA-based or chromium alloysbased, show some significant improvements in the treatment of CHD [78]. 
Regarding DES, Park et al., developed a polycaprolactone (PCL) 3D-printed sirolimus spray-coated drug eluting stent that was successfully tested in vivo, showing a sustained release profile and a reduced neointimal hyperplasia [79]. More recently, in order to substitute sirolimus with a newer drug, biolimus A9 (more lipophilic sirolimus derived drug) DES were produced [80]. Menown et al., tested the efficacy of a new biolimus A9 DES with cobalt chromium thin strut and poly-lactide acid (PLA) coating that exhibited low percentage of major adverse cardiac events (3.9\% at 9 months) and low rate of stent thrombosis (0.25\%) [81].

Stent's biocompatibility, mechanical properties and performances could be also improved via functionalisation of the strut and by coating their surface with active biomolecule. To cite an example, Zhou et al., created a new bioabsorbable stent made of Zinc $(\mathrm{Zn})$ and Copper $(\mathrm{Cu})$. It has been noticed that $\mathrm{Zn}$ positively influences growth and proliferation of endothelial cells and plays an important role in the prevention of cardiac diseases. To improve the stent's mechanical properties, $\mathrm{Cu}$ was added to the formulation, which induce the expression of vascular-endothelial growth factor (VEGF). To asses stent's properties and safety, the device was implanted in porcine coronary artery for 24 months and obtained very significant results, such as proper degradation rate, good endothelialisation process and no signs of thrombosis, necrosis or inflammatory response (Fig.5a) [82]. Another device is the one produced by Lee $e$ t al., in which, again, the benefits of 3D printing were exploited to create a personalized stent. The strut was made of PLA biodegradable polymer and then heparin-coated, aiming to improve stent's biocompatibility and prevent thrombosis. The device showed excellent anti-coagulant activity as well as modulation of smooth muscle cells and endothelial cells proliferation. In vivo tests resulted in less neointimal hyperplasia and no atherosclerosis and thrombosis (Fig.5b)[83]. Error! Reference source not found. summarise the main features of BMSs and DESs described in this section. 
All these mentioned devices can be useful tools in terms of treatment, but they do not allow for the monitoring of bodily parameters and prediction of up-coming pathological events. Microelectromechanical systems (MEMS) can be very useful for monitoring and prediction (e.g. blood pressure, temperature and chemical concentration of molecules) and for medication (e.g. delivering drugs in specific times) [84]. MEMS (bioMEMS when are used in biomedical applications) are miniaturized systems that consist in microelectronics components, micro actuators and microsensor [85, 86]. They allow the integration of multiple functions within the same device and to precisely control the drug delivery process thanks to the interplay of microtechnology and electronic components [10]. As an example, a multifunctional, bioresorbable electronic stent was developed by Son et al. The device was equipped with flow and temperature sensors, as well as a nanoparticles-based anti-inflammatory system (to eliminate oxygenreactive spices generated by $\mathrm{PCI}$ ) and nanospheres-based, temperature-controlled drug delivery system. Moreover, the electronical stent was also provided with a memory cell and wireless communication system for blood flow pattern analysis and diagnosis (Fig.6) [87].

\section{Diabetes}

CVDs, especially CHD, are very closely connected to diabetes mellitus. These pathologies share some significant points, such as worsening with age and obesity, also, they are both characterized by an anomalous lipidic profile.

Diabetes is a risk factor for CVDs (CVDs death rates are 1.7 times higher among people with diabetes), mainly due to all the hyperglycaemia-related issues that primarily involve the vascular system and nerves [88]. In the next section will be given an overview of diabetes pathophysiology. Additionally, it will focus about new strategies to implement insulin therapy. 


\subsection{Diabetes Pathophysiology and epidemiology}

Glucagon and insulin are the most important hormones involved in glucose metabolism [89]. The first is secreted when plasma glucose concentration $\left(C_{g}\right)$ is low, while the second is released with the increasing of $C_{g}$ levels [90]. They are respectively produced from pancreatic $\alpha$ and $\beta$ cells [91]: glucagon allows to control glycogenolysis and gluconeogenesis in case of fasting, on the other hand, insulin allows to start glycolysis and glycogenesis in high presence of glucose $[92,93]$.

Diabetes Mellitus (DM), in combination with obesity, dyslipidaemia and blood pressure elevation, is also known as metabolic syndrome [94]. It results from a defect in $\beta$ cells functioning and so in insulin secretion [95].

Type I diabetes usually develops during childhood and it is characterised by a selfdestruction of pancreatic $\beta$ cells due to an auto-immune reaction, that eventually leads to a decrease level of produced insulin; type II diabetes is very common in the elders and in case of obesity and involves both inadequate insulin production and peripheral insulin resistance $[93,94]$.

Lack of insulin, as well as defections in its production, are the main causes for the arising of a chronic state of hyperglycaemia [93] and other pathologies. In physiological conditions, glucose is up taken by cells (adipocytes and myocytes) by means of glucose transporter 4 (GLUT-4), an insulin-dependent protein that resides in the intracellular compartment and activates just in presence of this specific hormone [96]. In diabetic patients, adipose and muscular tissues are not able anymore to up take the circulating glucose due to insulin deficiency or resistance, thus causing hyperglycaemia [93]. Moreover, reduced concentration of insulin and increasing concentration of counter regulative hormones, stimulate triglycerides braking down, thus increasing free fatty acids blood concentration and production of ketone bodies, causing ketoacidosis [97, 98]. 
In this environment, severe diseases can easily develop. High levels of advanced glycation-end products (AGEs), such as glycated haemoglobin (HbA1c) and glycated albumin (GA) [99], are responsible for endothelial and vascular dysfunction, prolonged inflammation [100], bone damages [101] and fragility [102] and impaired functions in the peripheral nerves [103]. Vascular and endothelial abnormalities causes atherosclerosis and thrombosis [104], neuropathy and retinopathy [105]. Neuropathy and damages in blood circulation, as well as chronic inflammation, are the main causes for diabetic foot [106]. Excess $C_{g}$ can lead to the activation of numerous metabolic pathways that can be detrimental for some types of cells [101], to glycosuria and renal dysfunctions [97].

Hyperglycaemia can occur both in the basal state (overnight and between meals), due to an increased hepatic glucose production, and in the prandial state (after a meal), due to glucose absorption in the gastro-intestinal tract [94]. Therefore, in order to diagnose diabetes, glucose levels in the bloodstream must be measured carefully and they need to be within specific ranges (seeError! Reference source not found.). Moreover, due to high presence of AGEs, HbA1c is very often used as glycaemic indicator as well as $\mathrm{C}_{\mathrm{g}}$ [107].

To date, about 422 million people worldwide have diabetes, with type II being the most common, and about 1.6 million deaths can be attributed to this chronic disease [109].

Diabetes treatments rely mainly on the use of insulin and anti-diabetic agents, such as sulfonylureas, glinides, biguanides, glitazones (thiazolidinediones) and R-glucosidase inhibitors [94]. Currently, four types of insulin are commercially available (rapid-, short, intermediate- and long-acting) [110] and are commonly administered via subcutaneous injection (e.g. insulin syringes, pens and insulin pumps) [110, 111]. Despite the several advantages, such as low cost and high absorption, the administration method along with its frequency, may be the cause of infections, pain, tissue damages and low patient compliance [110, 112]. Hence, new therapeutic approaches are of pressing need. Among them, transdermal and closed loop drug delivery systems have shown some promise. 


\subsection{Transdermal drug delivery systems: microneedles}

Microneedles (MNs) have been extensively studied as transdermal DDSs, because of their low coast, easy handling, painless administration and no need for any power supply [110]. MNs can be solid, used as a pre-treatment for pore formation in the skin; coated, to fast release a small amount of drug (release depends on the coated amount); dissolving, in which drug is released due to needle dissolution; hollow, provided with a channel for drug infusion; reservoir-based swelling, in which release is achieved thanks to the absorption of interstitial fluids and needles subsequent swelling (Fig.7) [110, 113].

Kim et al. designed a patch-free and dissolution-based powder-carrying microneedle system (PCM) for insulin delivery and treatment of diabetes mellitus. PCMs were made of carboxymethyl cellulose $(\mathrm{CMC})$ and fabricated by means of a polydimethylsiloxane (PDMS) mould and solvent-casting method. Insulin powder was encapsulated into the needle and covered with a CMC protective layer. Through a micropillar system, PMCs could be finally inserted into the skin (Fig.8). Pharmacokinetic studies were carried on aiming to evaluate the maximum drug concentration $\left(\mathrm{C}_{\max }\right)$, time to reach $\mathrm{C}_{\max }\left(\mathrm{T}_{\max }\right)$, bioavailability $(\mathrm{BA})$ and the area under the serum drug concentration versus time curve (AUC). Results exhibited a prolonged release for PCMs and higher $\mathrm{C}_{\max }$ with respect to common dissolving microneedles (DMNs). Furthermore, PCMs showed good biocompatibility and insulin stability, along with some improvements in comparison to DMNs. Particularly, PCMs did not account for drug solubility and degradation during the fabrication process, which is one of the major issues associated with the use of DMNs [114].

Dissolvable systems are highly dependent upon dissolution rate of the employed material, therefore, could not be suitable for fast administrations [115]. To overcome this issue, Economidou et al. use stereolithography (SLA) 3D printing and inkjet printing, for the manufacturing of insulin-sugar film coated MNs array with two different geometries 
(spear and pyramidal). Both in vitro and in vivo studies were performed showing controlled release rates for both microneedles arrays, with a maximum insulin plasma concentration of $59.9 \mu \mathrm{IU} / \mathrm{ml}$ reached in the first hour; insulin was constantly released for up to $4 \mathrm{~h}[115]$.

\subsection{Closed-loop drug delivery systems}

Closed-loop drug delivery systems (or artificial pancreas) are feedback-based systems able to continuously monitor glucose levels in the blood and to deliver the right amount of insulin according to patient's $C_{g}$ variations, thus allowing for personalized treatment [111]. Artificial pancreas can be grouped in three main categories: the first is algorithm-based, which includes the use of electronic components, the second is polymer-based, in which insulin carriers are used, and the third exploits the properties of modified insulin $[112,116]$.

The simplest algorithm-based closed loop system is made of monitoring element (such as biosensors) which usually exploits molecules or enzymes that have high affinity with glucose (as Glucose Oxidase) [116], a control system, which provides dosing instructions, and a delivery device like insulin pumps [117]. Biosensors can be made of one or more electrodes, which are able to transduce free electric charges, produced by chemical redox reactions, in an electric current that can be easily processed by the control system [116]. The latter has the final aim of translating the obtained raw data and modulate drug infusion in accordance with a pre-set algorithm [111, 116]. The control system, as well as the delivery device, are usually worn by the patient, therefore need to be small, lightweight, wireless and with a long life battery [116].

The FDA-approved MiniMed 670G insulin pump (Medtronic) is an example of algorithm-based, hybrid closed-loop system. It can work in to two different modes: manual mode and auto mode. In manual mode, the patient is required to manually insert insulin delivery settings; in auto mode, the device is provided with and automated 
insulin pump and a fourth-generation sensor (Guardian sensor 3, Medtronic) that evaluates glucose concentration in the bloodstream with great accuracy. The insulin pump is programmed with a new proportional-integral-derivative algorithm (PID) able to adjust insulin delivery according to both $\mathrm{Cg}$ and the delivery history, avoiding postprandial hyperglycaemia and hypoglycaemia. This device still requires calibration and some user interventions, even though less in respect to other commercial devices [118].

Regarding polymer-based insulin delivery systems, glucose sensitivity can be reached using three types of glucose-sensitive molecule: Glucose Oxidase (GOx), glucose binding proteins (such as concanavalin A) and phenylboronic acid (PBA) [119]. Glucose can be converted into gluconic acid through the following set of chemical reactions:

$$
\begin{gathered}
\beta-D-\text { glucose }+\mathrm{O}_{2} \rightarrow D-\text { gluconolactone }+\mathrm{H}_{2} \mathrm{O}_{2} \\
2 \mathrm{H}_{2} \mathrm{O}_{2} \rightarrow 2 \mathrm{H}_{2} \mathrm{O}+\mathrm{O}_{2} \\
\text { D-gluconolactone }+\mathrm{H}_{2} \mathrm{O} \rightarrow \text { Gluconic Acid }
\end{gathered}
$$

The first reaction is catalyse by GOx, while the other two are spontaneous [120].

According to this mechanism of action, GOx-based drug delivery systems can be $\mathrm{pH}$-sensitive (swellable), due to the production of gluconic acid, hypoxia-sensitive, due to $\mathrm{O}_{2}$ consumption, and $\mathrm{H}_{2} \mathrm{O}_{2}$-sensitive [119].

Yu et al. developed a MN patch containing insulin-loaded polymersome-based vesicles for transcutaneous administration. The device was made of poly (ethylene glycol) (PEG) and polyserine modified with 2-nitromidazole via a thioether moiety. Insulin was released by vesicles dissociation subsequently to $\mathrm{H}_{2} \mathrm{O}_{2}$ production or to arising of hypoxia conditions. The device efficacy was evaluated in mice in vivo models, showing its ability to regulate glucose level in the bloodstream for about 10h [121]. 
Concanavalin A (Con-A) is carbohydrate-binding protein that is gaining attention in diabetes treatment due to its high glucose specificity [122]. Yin et al. produced a closedloop drug delivery system constituted by insulin-loaded and Con-A-based microspheres, integrated into a chitosan scaffold. The scaffold served as mechanical support and protection. Insulin delivery was achieved by degradation of the implant and subsequent erosion-dependent leakage of the microspheres. Results showed good biocompatibility and scaffold's ability to maintain insulin bioactivity. The delivery of insulin was sustained for almost 12 days with low burst release [123].

Eventually, as well as Con-A, PBA is a glucose-binding molecule characterized by great stability, easy preparation and flexibility. It is often used in swelling-based, degradation-based and competition-based drug delivery systems, especially in form of nanoparticles [112].

\section{Wound Healing}

Peripheral neuropathy and arterial disease, as well as a chronic inflammatory state and impairment in cellular functions, are commonly associated with diabetes and are among the main causes for diabetic foot and lower extremities ulcers. Particularly, 50\% of the non-traumatic amputations of the lower limbs worldwide are caused by diabetes [124]. This last section will review some of the newest dressings for chronic wounds treatment.

\subsection{Wound Pathophysiology and epidemiology}

Skin is the largest organ in the body and acts as a protective barrier against external factors [125]. It Protects internal organs from several type of pathogens [125] and provides excellent regeneration properties [126]. Wound healing is the biological process through which skin can repair from damages and it involves the interplay of several types of cells, 
growth factors, cytokine and chemokine [126]. This process can be divided into four main phases: homeostasis, inflammation, proliferation and remodelling [127].

Homeostasis (0-7 hours) is characterized by platelets aggregation and consequent vasoconstriction, aiming to prevent blood loss. The exposure of the extracellular matrix (ECM) activates platelets that, subsequently, bind to the collagen filaments. To produce a blood clot and a provisional ECM for cells binding and movement, platelets start to secrete different types of proteins (e.g. fibronectin) and growth factors (e.g. plateletderived growth factor, transforming growth factor $\beta$ ) that are able to recall immune cells and fibroblasts. During inflammation phase (1-3 days), neutrophils and monocytes arrive on the injured site. Neutrophils start to produce oxygen reactive spices (ROS), in order to clean the wound, while monocytes differentiate into macrophages and phagocyte bacteria, dead cells, ECM residues and every sort of external agents. These immune cells secrete growth factors and chemokines that stimulate granular tissue formation. Particularly, macrophages-derived growth factors (e.g. platelet-derived, fibroblasts, interleukin I and VI, tumour necrosis factor $\alpha$ ) activate fibroblasts, endothelial and smooth muscle cells and improves collagen deposition and thus ECM formation. At the end of this phase, macrophages phagocyte neutrophils. Proliferation phase (4-21 days) starts with the formation of granular tissue. Fibroblasts, keratinocytes and endothelial cells are recruited in the wound site to create new matrix and repair the damage.

Fibroblasts produce collagen type III and all the other components of the ECM (such as glycosaminoglycan's and proteoglycans), but also metalloproteins that have the specific task of degrading the provisional ECM. Furthermore, some fibroblasts differentiate into myofibroblasts and pull the wound edges in a process called wound contraction. Keratinocytes are recruited from the hair follicles and from the wound's boundaries towards the middle in order to start the re-epithelialization process. Eventually, endothelial cells, stimulated by the presence of vascular-endothelial growth factor (VEGF), start an angiogenetic process to build new blood vessels aiming to 
guarantee oxygen and nutrients supply to the growing tissue. In the remodelling phase (21 days - 1 year) fibroblasts and metalloproteins realign collagen fibres and convert collagen type III into collagen type I. At the end of the process, all the cells that are no longer needed exit from the injured site or undergo apoptosis. Finally, cells metabolic activity slow down and the new small vessels mature into larger vessels to complete angiogenesis [125-129].

In acute wounds the skin strictly follows this path, with the healing process taking different amount of time according to size, location and depth of the wound, patient's age and clinical history [127]. On the other hand, an impairment in this process could be the main cause for chronic wounds and hypertrophic scar/ keloid formation (abnormal and disorganized scar formation) [130-132]. Chronic wounds stall in the inflammation phase and are characterised by high levels of ROS, senescent cells and proinflammatory cytokines [127, 129], as well as an intense metalloproteins and bacterial activity (e.g. Staphylococcus Aureus, Pseudomonas Aeruginosa, Proteus Mirabilis, Escherichia Coli) with subsequent ECM excessive degradation and biofilm formation [133].

Burns and ulcers are very common types of chronic wounds. Burns are defined as any kind of chronic wounds caused by close and prolonged contact between the skin and a hot source, but also, as a consequence of the exposure to radioactive and corrosive materials [135]. Ulcers can form both on the outside of the body, especially in the lower extremities (e.g. venous ulcers, arterial ulcers, diabetic foot, pressure ulcers) [127, 136] and in the stomach (e.g. peptic ulcer) [137]. Vascular ulcers (e.g. venous and arterial) mainly develop because of venous/arterial fail in transporting blood, hypertension, atherosclerosis, vascular occlusion and subsequent ischemia [138, 139]. Diabetic foot ulcers are caused by hyperglycaemic-associated neuropathy, that progressively leads to a loss of protective sensation and improper ambulation, thus causing osteoarthropathy, skin dryness and tissue necrosis [140]. Pressure ulcers evolve due to prolonged exposure 
of the tissue to pressure values that exceed a certain tolerable threshold and that ultimately lead to ischemia, injuries and necrosis [141].

According to the World Health Organization (WHO), 180,000 deaths every year can be attributed to burns injuries while Gohel reported that around $0.3-1 \%$ of the western world population suffer from chronic leg ulceration [138]. Before starting any kind of treatment, patient assessments is crucial step in order to inspect the wound site and identify significative data of patient's clinical history [129, 142].

To date, clinical treatments mainly rely on debridement (e.g. surgical, mechanical, biological, enzymatic, autolytic), infection prevention strategies (e.g. antibiotic and antimicrobial agents administration, hyperbaric oxygen therapy) and biological therapy such as growth factors administration [134]. To protect the injured site from external agents and mechanical trauma, the wound is furthermore covered with dressings in dry, liquid or semi-solid composition [143].

\subsection{Wound dressings}

To be effective in the treatment of chronic wounds, the employed dressings must be biocompatible, protect the injured site from mechanical trauma and counteract bacterial infections; also, they should provide thermal insulation, absorb exudates, allow gas and liquid exchange and painless removal [136].

Generally, wound dressings can be divided into four main categories [136]:

- Passive dressings simply provide protection against bacteria and mechanical trauma;

- Interactive dressings allow gas exchange while acting as barrier;

- Advanced dressings can keep moist the wound bed;

- Bioactive dressings (e.g. for the delivery of bioactive compounds) play an important role in the healing process, thanks to their ability to guide and stimulate the tissue to self-regenerate. 
Error! Reference source not found. gives a general overview of the most common dressings used in wound management.

Gauze is the most widely employed and simple passive dressing that can be used in combination with other product and drugs. It is sterile, easily absorbs exudates and keep moist the wound bed. However, its removal is very often accompanied by secondary trauma [126].

Instead, the gold standard as bioactive dressing are grafts [145]. Autografts are autologous pieces of tissue withdrawn from the same patient and sub-cultured in order to obtain a larger cellular sheet that can be used to treat the wound [146]. Allografts and xenografts are decellularized pieces of dermis, the first usually obtained from human skin (e.g. Alloderm ${ }^{\circledR}$ (Life Cells Corporation)) [147], the latter from animal dermis (often porcine, ovine and bovine derived) [148]. Limitations associated with their use are mainly due to the poor availability (depending on the number of donors in the case of allografts), long processing time and high possibility of viral transmission $[145,148]$. To overcome these issues, new materials and new strategies are under investigation.

One of the biggest concern about wound healing is the bacterial colonization of the injured site, so antibiotics have been largely employed during the years, leading to an increasing tendency towards the development of antibiotic resistance [149]. Therefore, biologically derived polymers (e.g. chitosan or alginate) or substances (e.g. honey, essential oils or metal ions) with natural antimicrobial properties have been extensively studied $[149,150]$.

Metal ions have shown an antimicrobial behaviour and when used in combination with polymers are capable of altering the mechanical properties of the structure that are included, which can be very useful in the case of hydrogels. To improve the mechanical and antibacterial behaviour of alginate, Zhou et al. exploited the optimal characteristics of this material as wound dressing (in combination with polyacrylamide) and the ability of divalent ions (e.g. zinc, copper, strontium and calcium) to counteract 
infections. Their results showed that, among all the tested combinations, zinc-loaded scaffolds had the best antimicrobial performances against S. Aureus and E. Coli, plus they promoted wound closure, collagen deposition, granulation tissue formation and angiogenesis [151].

Vitexin is a plant-derived drug that showed some antibacterial, antiinflammatory and antiviral activities. Bektas et al. combined vitexin and chitosan to obtain a gel-based wound dressing which was able to improve the healing process and accelerate recovery. Tests were performed in both in vitro and in vivo, including cytotoxicity assay and wound healing assay, wound healing rat model and histopathological analysis. From the results, the synergistic activity of chitosan and vitexin was evident and this formulation significantly accelerated and improved the wound healing process, both in vitro and in vivo, with no cytotoxic effects [152]. Chitosan has also used in combination with MNs technology to create a chitosan microneedle array to deliver drugs and prevent infections. The device designed by Chi et al. was loaded with VEGF by means of the temperature-sensitive polymer poly( $\mathrm{N}$-isopropylacrylamide) (pNIPAM). It appeared from the drug release test that VEGF was successfully released from the device thanks to the smart behaviour of pNIPAM (that shrink at $37^{\circ} \mathrm{C}$, thus freeing the drug). Also, the device exhibited good antibacterial effects and promoted collagen deposition, granulation and vascularization (Fig.9) [153].

Among the new manufacturing techniques, electrospinning has been employed also in the production of wound dressings. Gorbani et al. combined the properties of different materials to produce an eletrcospun nanofibrous scaffold made of PCL, collagen and Zein loaded with zinc oxide ( $\mathrm{ZnO}$ ) nanoparticles (NPs) and Aloe Vera (Alv). Zein can provide collagen with antimicrobial activity, thermal durability and bioactive properties (e.g. cell adhesion, proliferation and penetration). The PCL increases mechanical stability, $\mathrm{ZnO}$ can confer further antibacterial properties to the implant and Alv can promote hydration, collagen synthesis, fibroblasts infiltration and accelerating 
the wound closure. They tested different Zein/PCL ratios, different collagen, $\mathrm{ZnO}$ and Alv concentrations; characterized the scaffold from a morphological and physicochemical point of view; performed mechanical tests, biological and drug delivery assays. Eventually, the optimal formulation was Zein/PCL (70:30) / collagen (20\% wt) / Alv $(8 \% \mathrm{wt}) / \mathrm{ZnO}(1 \% \mathrm{wt})$ and it proved to have good mechanical properties and excellent biocompatibility, as well as antibacterial activity and bioactivity [154].

Mancuso et al. have also used electrospinning for the development of an antibacterial mesh for soft tissue application by depositing layer-by-layer Manuka honey. The physicochemical characterization, biological and antibacterial tests showed that the addition of Manuka honey to the formulation did not alter the main characteristics of the scaffold and the layer-by-layer functionalization allowed a controlled release of the Manuka honey from the PCL electrospun fibres. Biological and antibacterial tests showed a good cytocompatibility and antimicrobial activity dependent on honey concentration and specific bacteria strain, in particular, activity against S. Aureus, E. Coli and P.Aeruginosa has been noticed [155].

Manuka Honey has been also employed with pectin and chitosan-propolis particles to give antibacterial, antiseptic, anti-inflammatory, anaesthetic effects to a 3Dprintable ink, that has been used by Andriotis et al. to print a bioactive patch [156]. 3D printing is an emerging technology that enables bioengineers to recreate complex $3 \mathrm{D}$ architectures in an easy and cost-effective way [157]. Wang et al. combined high voltage printing and 3D printing and designed a bi-layered membrane scaffold made of poly (lactic-co-glycolic acid) (PLGA) and alginate. The scaffold intended to recreate the bylayered structure of the skin, in which PLGA was used as the superior layer to mime the epidermis and alginate was used as the inferior layer to mime the dermis. The final implant provided a moist and insulated environment for the wound, promoted inflammation and stimulated vascularization and collagen deposition [158]. 
By means of $3 \mathrm{D}$ printing is also possible to encapsulate particles and drugs directly during the manufacturing process. Afghah et al. included silver nitrate particles in the formulation to provide antibacterial effects to a polycaprolactone-block-poly $(1,3-$ propylene succinate) (PCL-PPSu) 3D-printed scaffold. The implant's degradation and hydrophilicity carried on cytotoxicity assays as well as antibacterial tests against $S$. Aureus, C. Albicans, E. Coli, P. Aeruginosa. Especially concerning biological tests, results made it clear that the presence of silver nitrate $(1 \% \mathrm{wt} / \mathrm{wt})$ significantly enhanced the scaffold's antibacterial behaviour, being more effective for E. Coli and C. Albicans. Also, the inclusion of silver nitrate in the formulation did not alter the cytocompatibility of the used materials [159].

Another approach is based on the topical delivery of growth activators or inhibitors, such as growth factors and nucleic acids, aiming to regulate the production of specific proteins during the healing [160]. Mandapalli et al., fabricated a multi-layered polyelectrolyte film by means of layer-by-layer deposition of chitosan and alginate, including epidermal growth factor (EGF) and small interfering RNA (siRNA) in the formulation. Thanks to the gene silencing features of siRNA, the production of transforming growth factor $\beta$ (TGF- $\beta$ ), that is the main cause for hypertrophic scar formation, was inhibited. The device successfully improved the healing process enhancing re-epithelialization and controlling collagen deposition and scar formation [161].

It has been demonstrated that wounds can be sensitive to endogenous electric fields (EF), which is due to the presence of a natural electrical potential (transepithelial potential (TEP)) between the apical and the basal layer of the epithelium that is generated by the spatial and temporal variation of ions concentration and of ion channels distribution [162], [163]. When the skin is injured, TEP decreases and start to increase again, reaching its natural value, as soon as the healing process is completed [164]. Bhang et al. exploited this electrochemical phenomenon to manufacture a dermal piezoelectric- 
based patch able to generate an endogenous electric field. The movement-sensitive implant was made of PDMS and bidirectionally grown (BDG) zinc-oxide nanorods (NR) and it has been shown to promote wound healing and enhance cellular metabolism, migration and protein synthesis [165].

\section{Conclusions \& Expert Opinion}

Common drug administration methods, such as injection, oral or even nasal route, can usually be low-cost or easy ways to deliver drugs but, most of the times, they are not effective or are accompanied by some important drawbacks [110,113]. Injections can be used to quickly administer drugs in the systemic circulation, but hypodermic needles are not patient friendly [113]. Additionally, injected drugs, once administered, cannot be removed in case of adverse or allergic reaction [25]. Oral route is one of the most used, easiest and less invasive route of drug administration, but, before entering the hematic circulation, drugs bioavailability is often reduced due to first pass hepatic metabolism or degradation into the gastrointestinal (GI) tract $[113,166]$. Nasal delivery is a promising candidate to avoid liver metabolism, but there are still many challenges to overcome, such as mucociliary clearance, that could drastically reduce drug absorption [110]. In addition, another important aspect that has to be taken into account is patient adherence to the medical prescription. In some cases, to be effective drugs must be administered every day for long periods of time (as in the case of HIV and diabetes) and just one day of non-adherence can be extremely detrimental [7].

Long-acting implantable devices gained attention during the last few years to overcome all the issues connected to the old route of administration. Thanks to drug molecules encapsulated within the implants, bioavailability and stability of the formulation could be effectively preserved and improved due to implantation at some strategical sites, fist pass metabolism and GI degradation, as well as any compliancerelated problem are avoided. Their specific design allows for a precise and timely 
controlled drug release thus permitting a reduction of the administered dose. Additionally, implants can be removed in case of adverse reaction or drug exhaustion and in the case of biodegradable implants, there is no need for removal thanks to their ability to be absorbed from the body [4].

Lately, smart implantable devices have been successfully investigated, making the mechanism of drug release even more precise. These implants can release a specific amount of drug in response to the presence of a predetermined biomolecule thus eliminating every over or under-medication problem and medication adherence [116, 119].

Even if implantable devices could potentially revolutionize the drug administration process, they are subjected to some limitations and disadvantages. Implants still require invasive surgical operations to be inserted in the body and specialized personnel to perform implantation and explanation. These procedures, as well as the constant presence of a foreign object in the body, may cause patient pain and discomfort. Furthermore, their fabrication is way more expansive thus making difficult industrial-scale production [4].

Despite all these disadvantages, long-acting implantable devices are a very promising tool to allow a smart and effective drug administration. Many groups are carrying on research in this field, developing new devices using innovative technologies (e.g. 3D printing, Microfluidics, MEMS, electrospinning) and are investigating to overcome all the related limitations, using new materials, natural drugs and to make them smaller, more comfortable and patient-friendly, and implantable with a less invasive insertion procedure. Finally, many efforts have been focused on biodegradable materials in order to reach a better acceptance from the body and to avoid any further removal and surgical operation. 


\section{ACKNOWLEDGMENTS}

The authors would like to thank the University of Ulster for the Ph.D. scholarship to FC.

\section{CONFLICTS OF INTEREST}

The authors declare no conflict of interest.

\section{REFERENCES}

[1] P. Davoodi et al., "Drug delivery systems for programmed and on-demand release," Advanced Drug Delivery Reviews, vol. 132, pp. 104-138, Jul. 2018, doi: 10.1016/j.addr.2018.07.002.

[2] Y. Fu and W. J. Kao, "Drug release kinetics and transport mechanisms of nondegradable and degradable polymeric delivery systems," Expert Opinion on Drug Delivery, vol. 7, no. 4, pp. 429-444, Apr. 2010, doi: 10.1517/17425241003602259.

[3] S. T. Sanjay et al., "Recent advances of controlled drug delivery using microfluidic platforms," Advanced Drug Delivery Reviews, vol. 128, pp. 3-28, Mar. 2018, doi: 10.1016/j.addr.2017.09.013.

[4] L. W. Kleiner, J. C. Wright, and Y. Wang, "Evolution of implantable and insertable drug delivery systems," Journal of Controlled Release, vol. 181, pp. 1-10, May 2014, doi: 10.1016/j.jconrel.2014.02.006.

[5] U.S. Food and Drug Administration, "Implants and Prosthetics," FDA, Dec. 18, 2019. https://www.fda.gov/medical-devices/products-and-medicalprocedures/implants-and-prosthetics (accessed May 22, 2020). 
[6] R. James, U. S. Toti, S. G. Kumbar, and C. T. Laurencin, "Diseases and Clinical Applications that Can Benefit from Long Lasting Implants and Injections," in Long Acting Injections and Implants, J. C. Wright and D. J. Burgess, Eds. Boston, MA: Springer US, 2012, pp. 93-111.

[7] E. D. Weld and C. Flexner, "Long-acting implants to treat and prevent HIV infection:," Current Opinion in HIV and AIDS, vol. 15, no. 1, pp. 33-41, Jan. 2020, doi: 10.1097/COH.0000000000000591.

[8] J. Boateng and O. Catanzano, "Advanced Therapeutic Dressings for Effective Wound Healing-A Review," Journal of Pharmaceutical Sciences, vol. 104, no. 11, pp. 36533680, Nov. 2015, doi: 10.1002/jps.24610.

[9] L. Bally, H. Thabit, and R. Hovorka, "Glucose-responsive insulin delivery for type 1 diabetes: The artificial pancreas story," International Journal of Pharmaceutics, vol. 544, no. 2, pp. 309-318, Jun. 2018, doi: 10.1016/j.ijpharm.2017.12.022.

[10] H. J. Lee, N. Choi, E.-S. Yoon, and I.-J. Cho, "MEMS devices for drug delivery," Advanced Drug Delivery Reviews, vol. 128, pp. 132-147, Mar. 2018, doi: 10.1016/j.addr.2017.11.003.

[11] A. Mohammed, A. Elshaer, P. Sareh, M. Elsayed, and H. Hassanin, “Additive Manufacturing Technologies for Drug Delivery Applications," International Journal of Pharmaceutics, vol. 580, p. 119245, Apr. 2020, doi: 10.1016/j.ijpharm.2020.119245.

[12] I. Llorente García and M. Marsh, "A biophysical perspective on receptor-mediated virus entry with a focus on HIV," Biochimica et Biophysica Acta (BBA) - Biomembranes, vol. 1862, no. 6, p. 183158, Jun. 2020, doi: 10.1016/j.bbamem.2019.183158. 
[13] F. Xavier RUIZ and E. Arnold, "Evolving understanding of HIV-1 reverse transcriptase structure, function, inhibition, and resistance," Current Opinion in Structural Biology, vol. 61, pp. 113-123, Apr. 2020, doi: 10.1016/j.sbi.2019.11.011.

[14] J. A. Levy, "Pathogenesis of Human Immunodeficiency Virus Infection," MICROBIOL. REV., vol. 57, p. 107, 1993.

[15] G. Doitsh et al., "Cell death by pyroptosis drives CD4 T-cell depletion in HIV-1 infection," Nature, vol. 505, no. 7484, pp. 509-514, Jan. 2014, doi: 10.1038/nature12940.

[16] S. Carinelli, C. Xufré Ballesteros, M. Martí, S. Alegret, and M. I. Pividori, “Electrochemical magneto-actuated biosensor for CD4 count in AIDS diagnosis and monitoring," Biosensors and Bioelectronics, vol. 74, pp. 974-980, Dec. 2015, doi: 10.1016/j.bios.2015.07.053.

[17] T. Elliott et al., "Challenges of HIV diagnosis and management in the context of preexposure prophylaxis (PrEP), post-exposure prophylaxis (PEP), test and start and acute HIV infection: a scoping review," J Intern AIDS Soc, vol. 22, no. 12, Dec. 2019, doi: 10.1002/jia2.25419.

[18] N. Phanuphak and R. M. Gulick, "HIV treatment and prevention 2019: current standards of care," Current Opinion in HIV and AIDS, vol. 15, no. 1, pp. 4-12, Jan. 2020, doi: 10.1097/COH.0000000000000588.

[19] V. K. Sashindran and R. Chauhan, "Antiretroviral therapy: Shifting sands," Medical Journal Armed Forces India, vol. 72, no. 1, pp. 54-60, Jan. 2016, doi: 10.1016/j.mjafi.2015.12.002. 
[20] P. A. Volberding and S. G. Deeks, "Antiretroviral therapy and management of HIV infection," The Lancet, vol. 376, no. 9734, pp. 49-62, Jul. 2010, doi: 10.1016/S01406736(10)60676-9.

[21] R. M. Gulick and C. Flexner, "Long-Acting HIV Drugs for Treatment and Prevention," Annu. Rev. Med., vol. 70, no. 1, pp. 137-150, Jan. 2019, doi: 10.1146/annurev-med-041217-013717.

[22] F. P. Pons-Faudoa et al., “2-Hydroxypropyl- $\beta$-cyclodextrin-enhanced pharmacokinetics of cabotegravir from a nanofluidic implant for HIV pre-exposure prophylaxis," Journal of Controlled Release, vol. 306, pp. 89-96, Jul. 2019, doi: 10.1016/j.jconrel.2019.05.037.

[23] World Health Organization, "HIV/AIDS," WHO. https://www.who.int/westernpacific/health-topics/hiv-aids (accessed May 22, 2020).

[24] M. R. Sampson, S. B. Troy, Y. Belew, V. Arya, and K. A. Struble, “Considerations and challenges in developing novel long-acting antiretrovirals modalities for treatment and prevention of HIV-1 infection: a regulatory perspective," Current Opinion in HIV and AIDS, vol. 15, no. 1, pp. 61-65, Jan. 2020, doi: 10.1097/COH.0000000000000587.

[25] C. Flexner, "Antiretroviral implants for treatment and prevention of HIV infection:," Current Opinion in HIV and AIDS, vol. 13, no. 4, pp. 374-380, Jul. 2018, doi: 10.1097/COH.0000000000000470.

[26] R. D'Amico and D. A. Margolis, "Long-acting injectable therapy: an emerging paradigm for the treatment of HIV infection," vol. 15, no. 1, p. 6, 2019. 
[27] M. Kovarova et al., "Ultra-long-acting removable drug delivery system for HIV treatment and prevention," Nat Commun, vol. 9, no. 1, p. 4156, Dec. 2018, doi: 10.1038/s41467-018-06490-w.

[28] A. A. de Marchi et al., "Searching for a long-acting injectable formulation for the antiretroviral dolutegravir," British Journal of Pharmacy, vol. 4, no. 2, Dec. 2019, doi: 10.5920/bjpharm.568.

[29] K. Singh, S. G. Sarafianos, and A. Sönnerborg, "Long-Acting Anti-HIV Drugs Targeting HIV-1 Reverse Transcriptase and Integrase," Pharmaceuticals, vol. 12, no. 2, p. 62, Apr. 2019, doi: 10.3390/ph12020062.

[30] L. E. Coelho, T. S. Torres, V. G. Veloso, R. J. Landovitz, and B. Grinsztejn, “Preexposure prophylaxis 2.0: new drugs and technologies in the pipeline," The Lancet HIV, vol. 6, no. 11, pp. e788-e799, Nov. 2019, doi: 10.1016/S2352-3018(19)30238-3.

[31] M. E. Clement, R. Kofron, and R. J. Landovitz, “Long-acting injectable cabotegravir for the prevention of HIV infection:," Current Opinion in HIV and AIDS, vol. 15, no. 1, pp. 19-26, Jan. 2020, doi: 10.1097/COH.0000000000000597.

[32] M. Grobben, R. A. Stuart, and M. J. van Gils, “The potential of engineered antibodies for HIV-1 therapy and cure," Current Opinion in Virology, vol. 38, pp. 70-80, Oct. 2019, doi: 10.1016/j.coviro.2019.07.007.

[33] R. Gautam et al., "A single injection of crystallizable fragment domain-modified antibodies elicits durable protection from SHIV infection," Nat Med, vol. 24, no. 5, pp. 610-616, May 2018, doi: 10.1038/s41591-018-0001-2.

[34] C. Zhao et al., "Pharmacokinetics and Preliminary Safety of Pod-Intravaginal Rings Delivering the Monoclonal Antibody VRC01-N for HIV Prophylaxis in a Macaque 
Model," Antimicrob. Agents Chemother., vol. 61, no. 7, pp. e02465-16, /aac/61/7/e02465-16.atom, Jul. 2017, doi: 10.1128/AAC.02465-16.

[35] L. Gama and R. A. Koup, “New-Generation High-Potency and Designer Antibodies: Role in HIV-1 Treatment," Annu. Rev. Med., vol. 69, no. 1, pp. 409-419, Jan. 2018, doi: 10.1146/annurev-med-061016-041032.

[36] B. Julg and D. H. Barouch, "Neutralizing antibodies for HIV-1 prevention:," Current Opinion in HIV and AIDS, vol. 14, no. 4, pp. 318-324, Jul. 2019, doi: 10.1097/COH.0000000000000556.

[37] M. Caskey, "Broadly neutralizing antibodies for the treatment and prevention of HIV infection:," Current Opinion in HIV and AIDS, vol. 15, no. 1, pp. 49-55, Jan. 2020, doi: 10.1097/COH.0000000000000600.

[38] T. Palanee-Phillips and J. M. Baeten, "Topical delivery of long-acting antiretrovirals to prevent HIV acquisition:," Current Opinion in HIV and AIDS, vol. 15, no. 1, pp. $42-$ 48, Jan. 2020, doi: 10.1097/COH.0000000000000598.

[39] J. A. Moss et al., "Combination Pod-Intravaginal Ring Delivers Antiretroviral Agents for HIV Prophylaxis: Pharmacokinetic Evaluation in an Ovine Model," Antimicrob. Agents Chemother., vol. 60, no. 6, pp. 3759-3766, Jun. 2016, doi: 10.1128/AAC.00391-16.

[40] S. R. Ugaonkar et al., "A novel intravaginal ring to prevent HIV-1, HSV-2, HPV, and unintended pregnancy," Journal of Controlled Release, vol. 213, pp. 57-68, Sep. 2015, doi: 10.1016/j.jconrel.2015.06.018. 
[41] A. K. Blakney, Y. Jiang, and K. A. Woodrow, "Application of electrospun fibers for female reproductive health," Drug Deliv. and Transl. Res., vol. 7, no. 6, pp. 796-804, Dec. 2017, doi: 10.1007/s13346-017-0386-3.

[42] T. J. Sill and H. A. von Recum, "Electrospinning: Applications in drug delivery and tissue engineering," Biomaterials, vol. 29, no. 13, pp. 1989-2006, May 2008, doi: 10.1016/j.biomaterials.2008.01.011.

[43] T. N. Grooms et al., "Griffithsin-Modified Electrospun Fibers as a Delivery Scaffold To Prevent HIV Infection," Antimicrob. Agents Chemother., vol. 60, no. 11, pp. 65186531, Nov. 2016, doi: 10.1128/AAC.00956-16.

[44] L. M. Johnson et al., "Characterization of a Reservoir-Style Implant for Sustained Release of Tenofovir Alafenamide (TAF) for HIV Pre-Exposure Prophylaxis (PrEP)," Pharmaceutics, vol. 11, no. 7, p. 315, Jul. 2019, doi: 10.3390/pharmaceutics11070315.

[45] C. Y. X. Chua et al., "Transcutaneously refillable nanofluidic implant achieves sustained level of tenofovir diphosphate for HIV pre-exposure prophylaxis," Journal of Controlled Release, vol. 286, pp. 315-325, Sep. 2018, doi: 10.1016/j.jconrel.2018.08.010.

[46] P. Abgrall and N. T. Nguyen, "Nanofluidic Devices and Their Applications," Anal. Chem., vol. 80, no. 7, pp. 2326-2341, Apr. 2008, doi: 10.1021/ac702296u.

[47] K. So-Armah et al., "HIV and cardiovascular disease," The Lancet HIV, vol. 7, no. 4, pp. e279-e293, Apr. 2020, doi: 10.1016/S2352-3018(20)30036-9. 
[48] F. Sanchis-Gomar, C. Perez-Quilis, R. Leischik, and A. Lucia, "Epidemiology of coronary heart disease and acute coronary syndrome," Ann. Transl. Med., vol. 4, no. 13, pp. 256-256, Jul. 2016, doi: 10.21037/atm.2016.06.33.

[49] L. M. Buja, G. Ottaviani, and R. N. Mitchell, "Pathobiology of cardiovascular diseases: an update," Cardiovascular Pathology, vol. 42, pp. 44-53, Sep. 2019, doi: 10.1016/j.carpath.2019.06.002.

[50] “Cardiovascular Risk Factors. Insights From Framingham Heart Study | Revista Española de Cardiología (English Edition)." https://www.revespcardiol.org/encardiovascular-risk-factors-insights-from-articulo-13117552 (accessed Feb. 20, 2020).

[51] World Health Organization, "Cardiovascular diseases." https://www.who.int/westernpacific/health-topics/cardiovascular-diseases (accessed May 22, 2020).

[52] T. J. Guzik et al., "COVID-19 and the cardiovascular system: implications for risk assessment, diagnosis, and treatment options," Cardiovascular Research, p. cvaa106, Apr. 2020, doi: 10.1093/cvr/cvaa106.

[53] G. Aggarwal et al., "Association of Cardiovascular Disease With Coronavirus Disease 2019 (COVID-19) Severity: A Meta-Analysis," Current Problems in Cardiology, p. 100617, Apr. 2020, doi: 10.1016/j.cpcardiol.2020.100617.

[54] R. P. Lennon, K. A. Claussen, and K. A. Kuersteiner, "State of the Heart," Primary Care: Clinics in Office Practice, vol. 45, no. 1, pp. 1-15, Mar. 2018, doi: 10.1016/j.pop.2017.11.001. 
[55] P. H. Wirtz and R. von Känel, "Psychological Stress, Inflammation, and Coronary Heart Disease," Curr Cardiol Rep, vol. 19, no. 11, p. 111, Nov. 2017, doi: 10.1007/s11886-017-0919-x.

[56] A. Timmis, "Acute coronary syndromes," BMJ, p. h5153, Oct. 2015, doi: 10.1136/bmj.h5153.

[57] N. Makki, T. M. Brennan, and S. Girotra, "Acute Coronary Syndrome," J Intensive Care Med, vol. 30, no. 4, pp. 186-200, May 2015, doi: 10.1177/0885066613503294.

[58] M. J. Feinstein et al., "Patterns of Cardiovascular Mortality for HIV-Infected Adults in the United States: 1999 to 2013," The American Journal of Cardiology, vol. 117, no. 2, pp. 214-220, Jan. 2016, doi: 10.1016/j.amjcard.2015.10.030.

[59] M. Smit et al., "Future challenges for clinical care of an ageing population infected with HIV: a modelling study," The Lancet Infectious Diseases, vol. 15, no. 7, pp. 810818, Jul. 2015, doi: 10.1016/S1473-3099(15)00056-0.

[60] P. Y. Hsue and D. D. Waters, "HIV infection and coronary heart disease: mechanisms and management," Nat Rev Cardiol, vol. 16, no. 12, pp. 745-759, Dec. 2019, doi: 10.1038/s41569-019-0219-9.

[61] J. Moriya, "Critical roles of inflammation in atherosclerosis," Journal of Cardiology, vol. 73, no. 1, pp. 22-27, Jan. 2019, doi: 10.1016/j.jjcc.2018.05.010.

[62] K. S. Hongyu Li, J. H. Ruiping Zhao, F. W. Zhiru Hao, F. L. Yaojun Lu, and Yong Zhang, "Inflammatory biomarkers of coronary heart disease." https://www.ncbi.nlm.nih.gov/pubmed/28930526 (accessed Feb. 18, 2020).

[63] C. Bernelli et al., "Cardiovascular Events Recurrence and Coronary Artery Disease in HIV Patients: The Price We Have to Pay for the Chronicization of the Disease," 
Canadian Journal of Cardiology, vol. 36, no. 1, pp. 127-134, Jan. 2020, doi: 10.1016/j.cjca.2019.07.636.

[64] S. Torii et al., "Drug-eluting coronary stents: insights from preclinical and pathology studies," Nat Rev Cardiol, vol. 17, no. 1, pp. 37-51, Jan. 2020, doi: 10.1038/s41569-0190234-x.

[65] P. Majewska, E. Oledzka, and M. Sobczak, "Overview of the latest developments in the field of drug-eluting stent technology," Biomater. Sci., vol. 8, no. 2, pp. 544-551, 2020, doi: 10.1039/C9BM00468H.

[66] J. Y. Moon, F. Franchi, F. Rollini, and D. J. Angiolillo, “Evolution of Coronary Stent Technology and Implications for Duration of Dual Antiplatelet Therapy," Progress in Cardiovascular Diseases, vol. 60, no. 4-5, pp. 478-490, Jan. 2018, doi: 10.1016/j.pcad.2017.12.004.

[67] Y. J. Hong, H. Jeong, K. W. Cho, N. Lu, and D. Kim, “Wearable and Implantable Devices for Cardiovascular Healthcare: from Monitoring to Therapy Based on Flexible and Stretchable Electronics," Adv. Funct. Mater., vol. 29, no. 19, p. 1808247, May 2019, doi: 10.1002/adfm.201808247.

[68] L. E. L. Perkins and M. K. Rippy, "Balloons and Stents and Scaffolds: Preclinical Evaluation of Interventional Devices for Occlusive Arterial Disease," Toxicol Pathol, vol. 47, no. 3, pp. 297-310, Apr. 2019, doi: 10.1177/0192623318815604.

[69] D. Buccheri, D. Piraino, G. Andolina, and B. Cortese, “Understanding and managing in-stent restenosis: a review of clinical data, from pathogenesis to treatment," J. Thorac. Dis., vol. 8, no. 10, pp. E1150-E1162, Oct. 2016, doi: 10.21037/jtd.2016.10.93. 
[70] R. A. Byrne, M. Joner, and A. Kastrati, "Stent thrombosis and restenosis: what have we learned and where are we going? The Andreas Grüntzig Lecture ESC 2014," Eur Heart J, vol. 36, no. 47, pp. 3320-3331, Dec. 2015, doi: 10.1093/eurheartj/ehv511.

[71] K. Buchanan, A. Steinvil, and R. Waksman, "Does the new generation of drugeluting stents render bare metal stents obsolete?," Cardiovascular Revascularization Medicine, vol. 18, no. 6, pp. 456-461, Sep. 2017, doi: 10.1016/j.carrev.2017.03.027.

[72] H. M. Burt and W. L. Hunter, "Drug-eluting stents: A multidisciplinary success story," Advanced Drug Delivery Reviews, vol. 58, no. 3, pp. 350-357, Jun. 2006, doi: 10.1016/j.addr.2006.01.014.

[73] R. A. Partida and R. W. Yeh, "Contemporary Drug-Eluting Stent Platforms," Cardiology Clinics, vol. 35, no. 2, pp. 281-296, May 2017, doi: 10.1016/j.ccl.2016.12.010.

[74] A. S. Puranik, E. R. Dawson, and N. A. Peppas, "Recent advances in drug eluting stents," International Journal of Pharmaceutics, vol. 441, no. 1-2, pp. 665-679, Jan. 2013, doi: 10.1016/j.ijpharm.2012.10.029.

[75] D. S. Park et al., "Anti-restenotic and anti-thrombotic effect of polymer-free N-TiO2 film-based tacrolimus-eluting stent in a porcine model," Materials Today Communications, vol. 22, p. 100777, Mar. 2020, doi: 10.1016/j.mtcomm.2019.100777.

[76] W. Khan, S. Farah, and A. J. Domb, “Drug eluting stents: Developments and current status," Journal of Controlled Release, vol. 161, no. 2, pp. 703-712, Jul. 2012, doi: 10.1016/j.jconrel.2012.02.010.

[77] W. A. Omar and D. J. Kumbhani, "The Current Literature on Bioabsorbable Stents: a Review," Curr Atheroscler Rep, vol. 21, no. 12, p. 54, Dec. 2019, doi: 10.1007/s11883019-0816-4. 
[78] J. Nogic et al., "Biodegradable-Polymer Versus Polymer-Free Drug-Eluting Stents for the Treatment of Coronary Artery Disease," Cardiovascular Revascularization Medicine, vol. 20, no. 10, pp. 865-870, Oct. 2019, doi: 10.1016/j.carrev.2018.12.010.

[79] S. A. Park et al., "In vivo evaluation and characterization of a bio-absorbable drugcoated stent fabricated using a 3D-printing system," Materials Letters, vol. 141, pp. 355-358, Feb. 2015, doi: 10.1016/j.matlet.2014.11.119.

[80] R. A. Costa et al., "Angiographic Results of the First Human Experience With the Biolimus A9 Drug-Eluting Stent for De Novo Coronary Lesions," The American Journal of Cardiology, vol. 98, no. 4, pp. 443-446, Aug. 2006, doi: 10.1016/j.amjcard.2006.02.051.

[81] I. B. A. Menown et al., "First clinical evidence characterizing safety and efficacy of the new CoCr Biolimus-A9 eluting stent: The Biomatrix Alpha ${ }^{\mathrm{TM}}$ registry," IJC Heart E Vasculature, vol. 26, p. 100472, Feb. 2020, doi: 10.1016/j.ijcha.2020.100472.

[82] C. Zhou et al., "Long-term in vivo study of biodegradable Zn-Cu stent: A 2-year implantation evaluation in porcine coronary artery," Acta Biomaterialia, vol. 97, pp. 657-670, Oct. 2019, doi: 10.1016/j.actbio.2019.08.012.

[83] S. J. Lee et al., "Heparin coating on 3D printed poly (1-lactic acid) biodegradable cardiovascular stent via mild surface modification approach for coronary artery implantation," Chemical Engineering Journal, vol. 378, p. 122116, Dec. 2019, doi: 10.1016/j.cej.2019.122116.

[84] S. Chatterjee, M. Saxena, D. Padmanabhan, M. Jayachandra, and H. J. Pandya, "Futuristic medical implants using bioresorbable materials and devices," Biosensors and Bioelectronics, vol. 142, p. 111489, Oct. 2019, doi: 10.1016/j.bios.2019.111489. 
[85] A. C. R. Grayson et al., "A BioMEMS Review: MEMS Technology for Physiologically Integrated Devices," Proc. IEEE, vol. 92, no. 1, pp. 6-21, Jan. 2004, doi: 10.1109/JPROC.2003.820534.

[86] M. Bao and W. Wang, "Future of microelectromechanical systems (MEMS)," Sensors and Actuators A: Physical, vol. 56, no. 1-2, pp. 135-141, Aug. 1996, doi: 10.1016/09244247(96)01274-5.

[87] D. Son et al., "Bioresorbable Electronic Stent Integrated with Therapeutic Nanoparticles for Endovascular Diseases," ACS Nano, vol. 9, no. 6, pp. 5937-5946, Jun. 2015, doi: 10.1021/acsnano.5b00651.

[88] B. M. Leon, "Diabetes and cardiovascular disease: Epidemiology, biological mechanisms, treatment recommendations and future research," WJD, vol. 6, no. 13, p. 1246, 2015, doi: 10.4239/wjd.v6.i13.1246.

[89] J. E. Gerich, M. A. Charles, and G. M. Grodsky, "Regulation of Pancreatic Insulin and Glucagon Secretion," Annu. Rev. Physiol., vol. 38, no. 1, pp. 353-388, Mar. 1976, doi: 10.1146/annurev.ph.38.030176.002033.

[90] V. Grubelnik, J. Zmazek, R. Markovič, M. Gosak, and M. Marhl, “Modelling of energy-driven switch for glucagon and insulin secretion," Journal of Theoretical Biology, vol. 493, p. 110213, May 2020, doi: 10.1016/j.jtbi.2020.110213.

[91] R. H. Unger, "Glucagon Physiology and Pathophysiology," http://dx.doi.org/10.1056/NEJM197108192850806, Jan. $14, \quad 2010$. https://www.nejm.org/doi/pdf/10.1056/NEJM197108192850806 (accessed Apr. 08, 2020). 
[92] S. L. Aronoff, K. Berkowitz, B. Shreiner, and L. Want, "Glucose Metabolism and Regulation: Beyond Insulin and Glucagon," Diabetes Spectrum, vol. 17, no. 3, pp. 183-190, Jul. 2004, doi: 10.2337/diaspect.17.3.183.

[93] J. Moini, "Pathophysiology of Diabetes," in Epidemiology of Diabetes, Elsevier, 2019, pp. 25-43.

[94] J. S. Skyler, "Diabetes Mellitus: Pathogenesis and Treatment Strategies," J. Med. Chem., vol. 47, no. 17, pp. 4113-4117, Aug. 2004, doi: 10.1021/jm0306273.

[95] A. D. Association, "Diagnosis and Classification of Diabetes Mellitus," Diabetes Care, vol. 28, no. suppl 1, pp. s37-s42, Jan. 2005, doi: 10.2337/diacare.28.suppl_1.S37.

[96] D. E. James, R. Brown, J. Navarro, and P. F. Pilch, "Insulin-regulatable tissues express a unique insulin-sensitive glucose transport protein," Nature, vol. 333, no. 6169, pp. 183-185, May 1988, doi: 10.1038/333183a0.

[97] E. A. Nyenwe and A. E. Kitabchi, "The evolution of diabetic ketoacidosis: An update of its etiology, pathogenesis and management," Metabolism, vol. 65, no. 4, pp. 507521, Apr. 2016, doi: 10.1016/j.metabol.2015.12.007.

[98] K. K. Dhatariya, "Defining and characterising diabetic ketoacidosis in adults," Diabetes Research and Clinical Practice, vol. 155, p. 107797, Sep. 2019, doi: 10.1016/j.diabres.2019.107797.

[99] T. Gan, X. Liu, and G. Xu, "Glycated Albumin Versus HbA1c in the Evaluation of Glycemic Control in Patients With Diabetes and CKD," Kidney International Reports, vol. 3, no. 3, pp. 542-554, May 2018, doi: 10.1016/j.ekir.2017.11.009. 
[100] N. Dulull, F. Kwa, N. Osman, U. Rai, B. Shaikh, and T. R. Thrimawithana, "Recent advances in the management of diabetic retinopathy," Drug Discovery Today, vol. 24, no. 8, pp. 1499-1509, Aug. 2019, doi: 10.1016/j.drudis.2019.03.028.

[101] D. E. Sellmeyer, R. Civitelli, L. C. Hofbauer, S. Khosla, B. Lecka-Czernik, and A. V. Schwartz, "Skeletal Metabolism, Fracture Risk, and Fracture Outcomes in Type 1 and Type 2 Diabetes," Diabetes, vol. 65, no. 7, pp. 1757-1766, Jul. 2016, doi: 10.2337/db16-0063.

[102] S. Lau and M. Lee, "Hyperglycaemia is an under-appreciated but modifiable risk factor in managing people with type 1 diabetes and fragility fractures," The Foot, vol. 40, pp. 43-45, Sep. 2019, doi: 10.1016/j.foot.2019.04.005.

[103] G. Sloan et al., "A new look at painful diabetic neuropathy," Diabetes Research and Clinical Practice, vol. 144, pp. 177-191, Oct. 2018, doi: 10.1016/j.diabres.2018.08.020.

[104] R. Retnakaran and B. Zinman, "Type 1 diabetes, hyperglycaemia, and the heart," The Lancet, vol. 371, no. 9626, pp. 1790-1799, May 2008, doi: 10.1016/S01406736(08)60767-9.

[105] I. Ahmad and M. Hoda, "Attenuation of diabetic retinopathy and neuropathy by resveratrol: Review on its molecular mechanisms of action," Life Sciences, vol. 245, p. 117350, Mar. 2020, doi: 10.1016/j.lfs.2020.117350.

[106] K. Wicks, T. Torbica, and K. A. Mace, "Myeloid cell dysfunction and the pathogenesis of the diabetic chronic wound," Seminars in Immunology, vol. 26, no. 4, pp. 341-353, Aug. 2014, doi: 10.1016/j.smim.2014.04.006. 
[107] M. Incani et al., "Glycated hemoglobin for the diagnosis of diabetes and prediabetes: Diagnostic impact on obese and lean subjects, and phenotypic characterization," $J$ Diabetes Invest, vol. 6, no. 1, pp. 44-50, Jan. 2015, doi: 10.1111/jdi.12241.

[108] A. M. Egan and S. F. Dinneen, “What is diabetes?," Medicine, vol. 47, no. 1, pp. 1-4, Jan. 2019, doi: 10.1016/j.mpmed.2018.10.002.

[109] World Health Organization, "Diabetes." https://www.who.int/westernpacific/health-topics/diabetes (accessed May 22, 2020).

[110] X. Jin, D. D. Zhu, B. Z. Chen, M. Ashfaq, and X. D. Guo, “Insulin delivery systems combined with microneedle technology," Advanced Drug Delivery Reviews, vol. 127, pp. 119-137, Mar. 2018, doi: 10.1016/j.addr.2018.03.011.

[111] K. Dovc and T. Battelino, "Evolution of Diabetes Technology," Endocrinology and Metabolism Clinics of North America, vol. 49, no. 1, pp. 1-18, Mar. 2020, doi: 10.1016/j.ecl.2019.10.009.

[112] Q. Huang, L. Wang, H. Yu, and K. Ur-Rahman, "Advances in phenylboronic acidbased closed-loop smart drug delivery system for diabetic therapy," Journal of Controlled Release, vol. 305, pp. 50-64, Jul. 2019, doi: 10.1016/j.jconrel.2019.05.029.

[113] Y.-C. Kim, J.-H. Park, and M. R. Prausnitz, "Microneedles for drug and vaccine delivery," Advanced Drug Delivery Reviews, vol. 64, no. 14, pp. 1547-1568, Nov. 2012, doi: 10.1016/j.addr.2012.04.005.

[114] S. Kim, H. Yang, J. Eum, Y. Ma, S. Fakhraei Lahiji, and H. Jung, "Implantable powder-carrying microneedles for transdermal delivery of high-dose insulin with 
enhanced activity," Biomaterials, vol. 232, p. 119733, Feb. 2020, doi: 10.1016/j.biomaterials.2019.119733.

[115] S. N. Economidou et al., "3D printed microneedle patches using stereolithography (SLA) for intradermal insulin delivery," Materials Science and Engineering: C, vol. 102, pp. 743-755, Sep. 2019, doi: 10.1016/j.msec.2019.04.063.

[116] K. Scholten and E. Meng, "A review of implantable biosensors for closed-loop glucose control and other drug delivery applications," International Journal of Pharmaceutics, vol. 544, no. 2, pp. 319-334, Jun. 2018, doi: 10.1016/j.ijpharm.2018.02.022.

[117] R. W. Beck, R. M. Bergenstal, L. M. Laffel, and J. C. Pickup, “Advances in technology for management of type 1 diabetes," The Lancet, vol. 394, no. 10205, pp. 1265-1273, Oct. 2019, doi: 10.1016/S0140-6736(19)31142-0.

[118] A. Saunders, L. H. Messer, and G. P. Forlenza, “MiniMed 670G hybrid closed loop artificial pancreas system for the treatment of type 1 diabetes mellitus: overview of its safety and efficacy," Expert Review of Medical Devices, vol. 16, no. 10, pp. 845-853, Oct. 2019, doi: 10.1080/17434440.2019.1670639.

[119] J. Yu, Y. Zhang, J. Yan, A. R. Kahkoska, and Z. Gu, "Advances in bioresponsive closed-loop drug delivery systems," International Journal of Pharmaceutics, vol. 544, no. 2, pp. 350-357, Jun. 2018, doi: 10.1016/j.ijpharm.2017.11.064.

[120] C. M. Wong, K. H. Wong, and X. D. Chen, “Glucose oxidase: natural occurrence, function, properties and industrial applications," Appl Microbiol Biotechnol, vol. 78, no. 6, pp. 927-938, Apr. 2008, doi: 10.1007/s00253-008-1407-4. 
[121] J. Yu et al., "Hypoxia and $\mathrm{H}_{2} \mathrm{O}_{2}$ Dual-Sensitive Vesicles for Enhanced GlucoseResponsive Insulin Delivery," Nano Lett., vol. 17, no. 2, pp. 733-739, Feb. 2017, doi: 10.1021/acs.nanolett.6b03848.

[122] R. Yin, M. Bai, J. He, J. Nie, and W. Zhang, “Concanavalin A-sugar affinity based system: Binding interactions, principle of glucose-responsiveness, and modulated insulin release for diabetes care," International Journal of Biological Macromolecules, vol. 124, pp. 724-732, Mar. 2019, doi: 10.1016/j.ijbiomac.2018.11.261.

[123] R. Yin et al., "Engineering synthetic artificial pancreas using chitosan hydrogels integrated with glucose-responsive microspheres for insulin delivery," Materials Science and Engineering: C, vol. 96, pp. 374-382, Mar. 2019, doi: 10.1016/j.msec.2018.11.032.

[124] S. Dangwal et al., "Impairment of Wound Healing in Patients With Type 2 Diabetes Mellitus Influences Circulating MicroRNA Patterns via Inflammatory Cytokines," Arterioscler Thromb Vasc Biol., vol. 35, no. 6, pp. 1480-1488, Jun. 2015, doi: 10.1161/ATVBAHA.114.305048.

[125] A. J. Singer, "Cutaneous Wound Healing," The New England Journal of Medicine, p. 9, 1999.

[126]S. Saghazadeh et al., "Drug delivery systems and materials for wound healing applications," Advanced Drug Delivery Reviews, vol. 127, pp. 138-166, Mar. 2018, doi: 10.1016/j.addr.2018.04.008.

[127] L. M. Morton and T. J. Phillips, "Wound healing and treating wounds," Journal of the American Academy of Dermatology, vol. 74, no. 4, pp. 589-605, Apr. 2016, doi: 10.1016/j.jaad.2015.08.068. 
[128] I. Hall Barrientos, G. MacKenzie, C. Wilson, D. Lamprou, and P. Coats, “Biological Performance of Electrospun Polymer Fibres," Materials, vol. 12, no. 3, p. 363, Jan. 2019, doi: 10.3390/ma12030363.

[129] R. G. Frykberg and J. Banks, "Challenges in the Treatment of Chronic Wounds," Advances in Wound Care, vol. 4, no. 9, pp. 560-582, Sep. 2015, doi: 10.1089/wound.2015.0635.

[130] B. A. Mast and G. S. Schultz, "Interactions of cytokines, growth factors, and proteases in acute and chronic wounds," Wound Rep. Reg., vol. 4, no. 4, pp. 411-420, Oct. 1996, doi: 10.1046/j.1524-475X.1996.40404.x.

[131] M. E. Okur, I. D. Karantas, Z. Şenyiğit, N. Üstündağ Okur, and P. I. Siafaka, “Recent trends on wound management: New therapeutic choices based on polymeric carriers," Asian Journal of Pharmaceutical Sciences, p. S1818087619311808, Jan. 2020, doi: 10.1016/j.ajps.2019.11.008.

[132] P.-H. Wang, B.-S. Huang, H.-C. Horng, C.-C. Yeh, and Y.-J. Chen, “Wound healing," Journal of the Chinese Medical Association, vol. 81, no. 2, pp. 94-101, Feb. 2018, doi: 10.1016/j.jcma.2017.11.002.

[133] V. R. Krishnaswamy, D. Mintz, and I. Sagi, "Matrix metalloproteinases: The sculptors of chronic cutaneous wounds," Biochimica et Biophysica Acta (BBA) Molecular Cell Research, vol. 1864, no. 11, pp. 2220-2227, Nov. 2017, doi: 10.1016/j.bbamcr.2017.08.003.

[134] H. S. Kim, X. Sun, J.-H. Lee, H.-W. Kim, X. Fu, and K. W. Leong, “Advanced drug delivery systems and artificial skin grafts for skin wound healing," Advanced Drug Delivery Reviews, vol. 146, pp. 209-239, Jun. 2019, doi: 10.1016/j.addr.2018.12.014. 
[135] M. Denzinger et al., "Hemocompatibility of different burn wound dressings," Wound Rep Reg, vol. 27, no. 5, pp. 470-476, Sep. 2019, doi: 10.1111/wrr.12739.

[136] M. Abrigo, S. L. McArthur, and P. Kingshott, “Electrospun Nanofibers as Dressings for Chronic Wound Care: Advances, Challenges, and Future Prospects: Electrospun Nanofibers as Dressings for Chronic Wound Care ...," Macromol. Biosci., vol. 14, no. 6, pp. 772-792, Jun. 2014, doi: 10.1002/mabi.201300561.

[137] R. T. Kavitt, A. M. Lipowska, A. Anyane-Yeboa, and I. M. Gralnek, “Diagnosis and Treatment of Peptic Ulcer Disease," The American Journal of Medicine, vol. 132, no. 4, pp. 447-456, Apr. 2019, doi: 10.1016/j.amjmed.2018.12.009.

[138] M. Gohel, “Chronic ulceration of the leg," Surgery (Oxford), vol. 37, no. 2, pp. 88-92, Feb. 2019, doi: 10.1016/j.mpsur.2018.12.009.

[139] J. A. Caprini, H. Partsch, and R. Simman, "Venous Ulcers," Journal of the American College of Clinical Wound Specialists, vol. 4, no. 3, pp. 54-60, Sep. 2012, doi: 10.1016/j.jccw.2013.11.001.

[140] C. A. Chastain, N. Klopfenstein, C. H. Serezani, and D. M. Aronoff, "A Clinical Review of Diabetic Foot Infections," Clinics in Podiatric Medicine and Surgery, vol. 36, no. 3, pp. 381-395, Jul. 2019, doi: 10.1016/j.cpm.2019.02.004.

[141] J. S. Mervis and T. J. Phillips, “Pressure ulcers: Pathophysiology, epidemiology, risk factors, and presentation," Journal of the American Academy of Dermatology, vol. 81, no. 4, pp. 881-890, Oct. 2019, doi: 10.1016/j.jaad.2018.12.069.

[142] K. Vowden and P. Vowden, "Wound dressings: principles and practice," Surgery (Oxford), vol. 35, no. 9, pp. 489-494, Sep. 2017, doi: 10.1016/j.mpsur.2017.06.005. 
[143] J. Wang and M. Windbergs, "Functional electrospun fibers for the treatment of human skin wounds," European Journal of Pharmaceutics and Biopharmaceutics, vol. 119, pp. 283-299, Oct. 2017, doi: 10.1016/j.ejpb.2017.07.001.

[144] V. Jones, J. E. Grey, and K. G. Harding, “Wound dressings,” BMJ, vol. 332, no. 7544, pp. 777-780, Apr. 2006.

[145] E. Axibal and M. Brown, "Surgical Dressings and Novel Skin Substitutes," Dermatologic Clinics, vol. 37, no. 3, pp. 349-366, Jul. 2019, doi: 10.1016/j.det.2019.03.005.

[146] J. M. Junkins-Hopkins, "Biologic dressings," Journal of the American Academy of Dermatology, vol. 64, no. 1, pp. e5-e7, Jan. 2011, doi: 10.1016/j.jaad.2010.09.729.

[147] N. P. Patel and A. Lawrence Cervino, "Keloid treatment: Is there a role for acellular human dermis (Alloderm)?," Journal of Plastic, Reconstructive \& Aesthetic Surgery, vol. 63, no. 8, pp. 1344-1348, Aug. 2010, doi: 10.1016/j.bjps.2009.07.032.

[148] T. Chiu and A. Burd, "'Xenograft' dressing in the treatment of burns," Clinics in Dermatology, vol. 23, no. 4, pp. 419-423, Jul. 2005, doi: 10.1016/j.clindermatol.2004.07.027.

[149] S. García-Salinas et al., "Electrospun anti-inflammatory patch loaded with essential oils for wound healing," International Journal of Pharmaceutics, vol. 577, p. 119067, Mar. 2020, doi: 10.1016/j.ijpharm.2020.119067.

[150] G. Schoukens, "Bioactive dressings to promote wound healing," in Advanced Textiles for Wound Care, Elsevier, 2019, pp. 135-167.

[151] Q. Zhou et al., "Influence of different divalent ions cross-linking sodium alginatepolyacrylamide hydrogels on antibacterial properties and wound healing," 
Carbohydrate Polymers, vol. 197, pp. 292-304, Oct. 2018, doi: 10.1016/j.carbpol.2018.05.078.

[152] N. Bektas, B. Şenel, E. Yenilmez, O. Özatik, and R. Arslan, “Evaluation of wound healing effect of chitosan-based gel formulation containing vitexin," Saudi Pharmaceutical Journal, vol. 28, no. 1, pp. 87-94, Jan. 2020, doi: 10.1016/j.jsps.2019.11.008.

[153] J. Chi, X. Zhang, C. Chen, C. Shao, Y. Zhao, and Y. Wang, "Antibacterial and angiogenic chitosan microneedle array patch for promoting wound healing," Bioactive Materials, vol. 5, no. 2, pp. 253-259, Jun. 2020, doi: 10.1016/j.bioactmat.2020.02.004.

[154] M. Ghorbani, P. Nezhad-Mokhtari, and S. Ramazani, "Aloe vera-loaded nanofibrous scaffold based on Zein/Polycaprolactone/Collagen for wound healing," International Journal of Biological Macromolecules, vol. 153, pp. 921-930, Jun. 2020, doi: 10.1016/j.ijbiomac.2020.03.036.

[155] E. Mancuso, C. Tonda-Turo, C. Ceresa, V. Pensabene, L. Fracchia, and P. Gentile, "Potential of Manuka Honey as a natural polyelectrolyte to develop biomimetic nanostructured meshes with antimicrobial properties," p. 28.

[156] E. G. Andriotis, G. K. Eleftheriadis, C. Karavasili, and D. G. Fatouros, “Development of Bio-Active Patches Based on Pectin for the Treatment of Ulcers and Wounds Using 3D-Bioprinting Technology," Pharmaceutics, vol. 12, no. 1, p. 56, Jan. 2020, doi: 10.3390/pharmaceutics12010056.

[157] W. Zhu, X. Ma, M. Gou, D. Mei, K. Zhang, and S. Chen, “3D printing of functional biomaterials for tissue engineering," Current Opinion in Biotechnology, vol. 40, pp. 103-112, Aug. 2016, doi: 10.1016/j.copbio.2016.03.014. 
[158] S. Wang et al., "Three Dimensional Printing Bilayer Membrane Scaffold Promotes Wound Healing," Front. Bioeng. Biotechnol., vol. 7, p. 348, Nov. 2019, doi: 10.3389/fbioe.2019.00348.

[159] F. Afghah et al., "3D printing of silver-doped polycaprolactone-poly propylene succinate composite scaffolds for skin tissue engineering," Biomed. Mater., Feb. 2020, doi: 10.1088/1748-605X/ab7417.

[160] Ö. Tezgel et al., "Collagen scaffold-mediated delivery of NLC/siRNA as wound healing materials," Journal of Drug Delivery Science and Technology, vol. 55, p. 101421, Feb. 2020, doi: 10.1016/j.jddst.2019.101421.

[161] P. K. Mandapalli et al., “Layer-by-Layer Thin Films for Co-Delivery of TGF- $\beta$ siRNA and Epidermal Growth Factor to Improve Excisional Wound Healing," AAPS PharmSciTech, vol. 18, no. 3, pp. 809-820, Apr. 2017, doi: 10.1208/s12249-016-0571-6.

[162] B. Song, Y. Gu, J. Pu, B. Reid, Z. Zhao, and M. Zhao, “Application of direct current electric fields to cells and tissues in vitro and modulation of wound electric field in vivo," Nat Protoc, vol. 2, no. 6, pp. 1479-1489, Jun. 2007, doi: 10.1038/nprot.2007.205.

[163] R. Nuccitelli, "A Role for Endogenous Electric Fields in Wound Healing," in Current Topics in Developmental Biology, vol. 58, Elsevier, 2003, pp. 1-26.

[164] J. Dubé et al., “Restoration of the Transepithelial Potential Within Tissue-Engineered Human Skin In Vitro and During the Wound Healing Process In Vivo," Tissue Engineering Part A, vol. 16, no. 10, pp. 3055-3063, Oct. 2010, doi: 10.1089/ten.tea.2010.0030. 
[165] S. H. Bhang et al., "Zinc Oxide Nanorod-Based Piezoelectric Dermal Patch for Wound Healing," Adv. Funct. Mater., vol. 27, no. 1, p. 1603497, Jan. 2017, doi: 10.1002/adfm.201603497.

[166] R. Singh, S. Singh, and J. W. Lillard, "Past, Present, and Future Technologies for Oral Delivery of Therapeutic Proteins," Journal of Pharmaceutical Sciences, vol. 97, no. 7, pp. 2497-2523, Jul. 2008, doi: 10.1002/jps.21183. 


\section{Figures Captions}

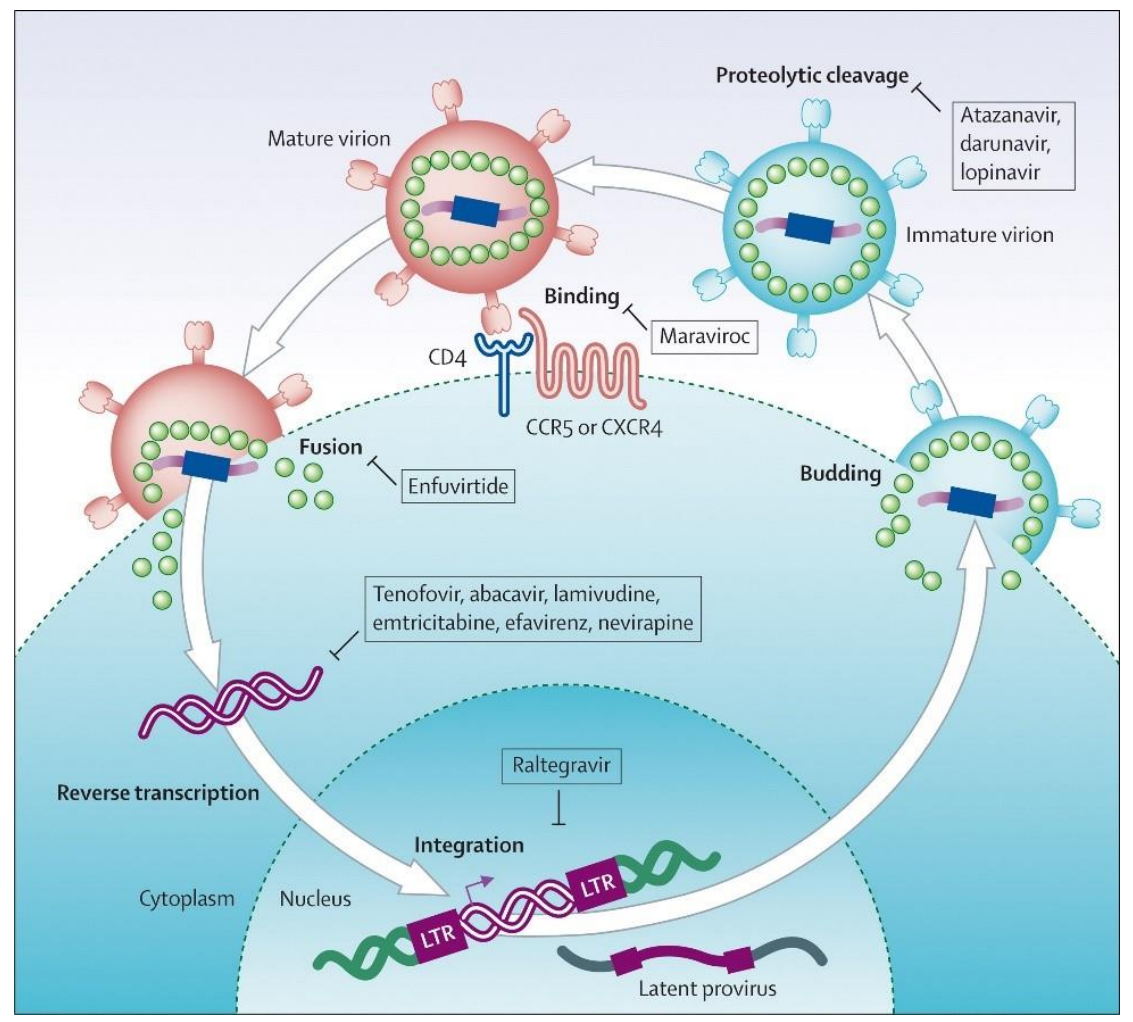

Fig. 1: Mechanism of action of some antiretroviral drugs [20].
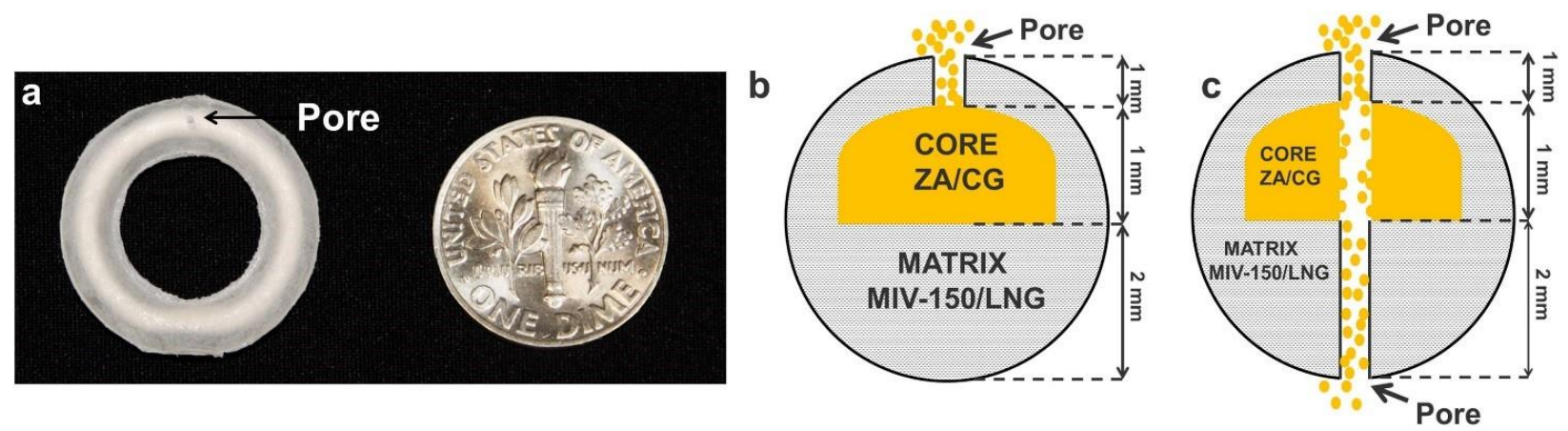

Fig. 2: Core matrix IVR, a) Photo of the device. b) and c) device cross sections [40]. 

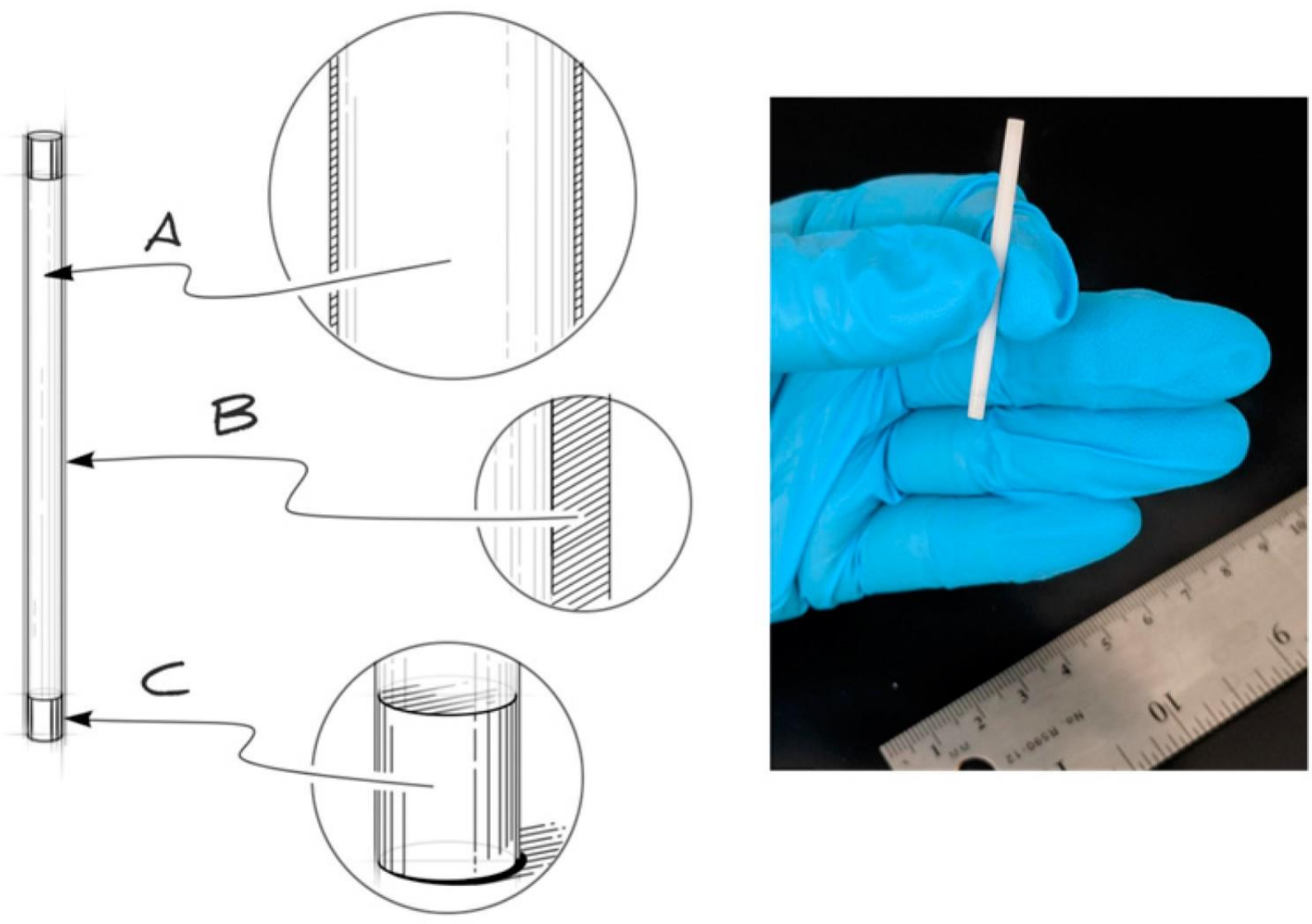

Fig. 3: Subcutaneous implant by Johnson et al. On the left, schematization of the device in which A represents the drug core, B the rate-controlling membrane and C PCL caps. On the right, photo of the same device [44]. 

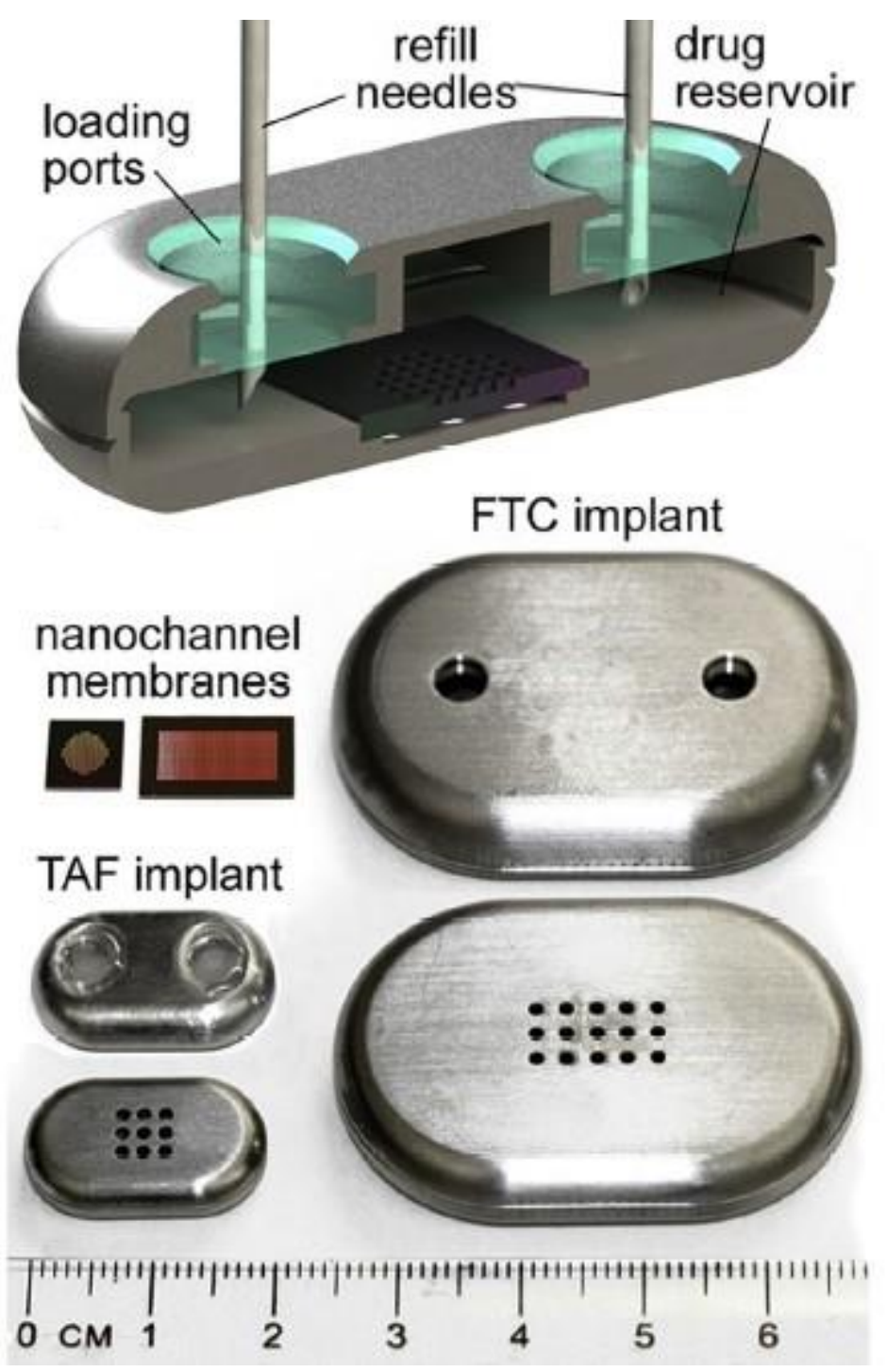

Fig. 4: Cross sectional, top and bottom view of refillable nanofluidic implantable device by Chua et al. (adapted [45]). 


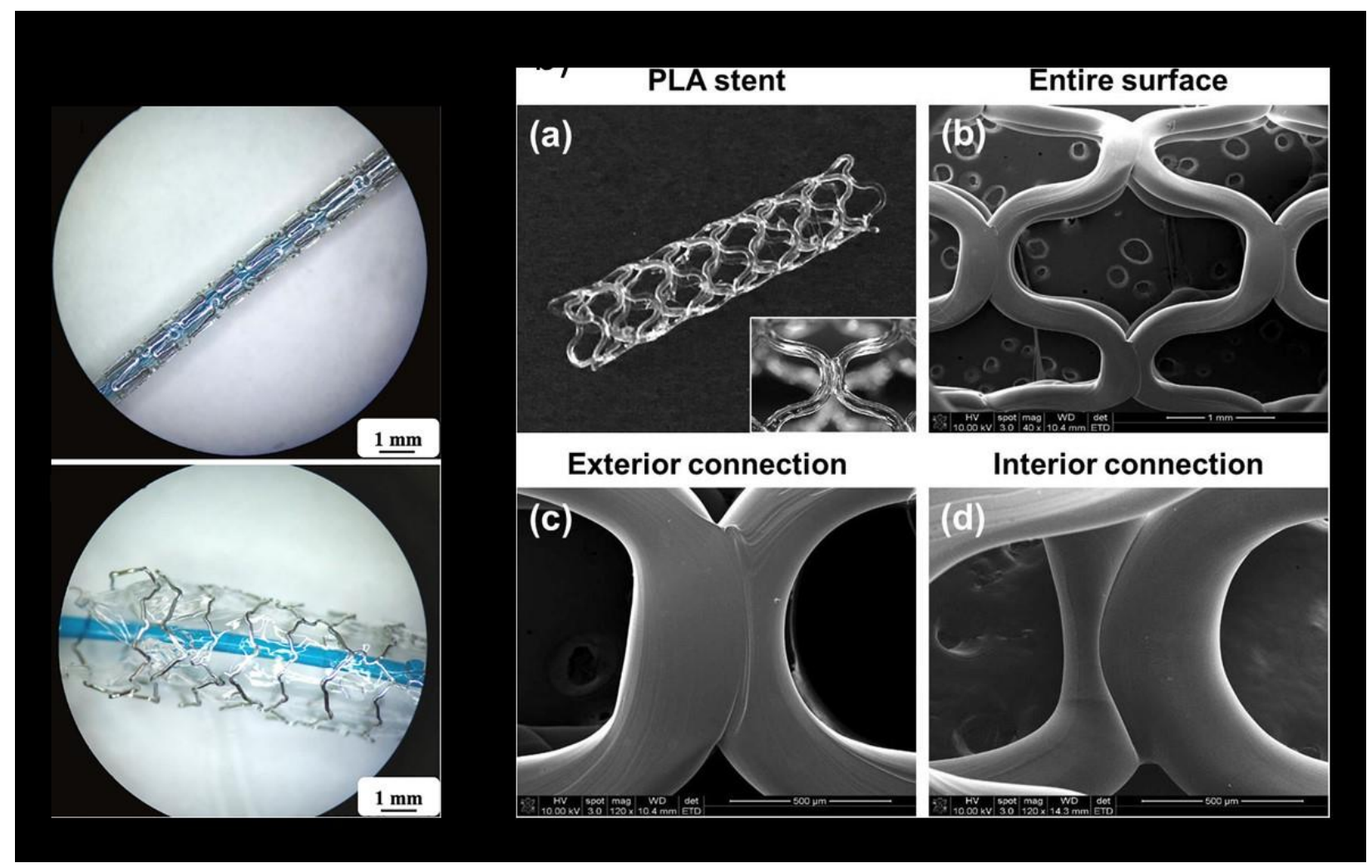

Fig. 5: (a) Zn-Cu biodegradable stent (adapted from [82]) (b) PLA 3D-printed heparincoated biodegradable stent (adapted from [83]).

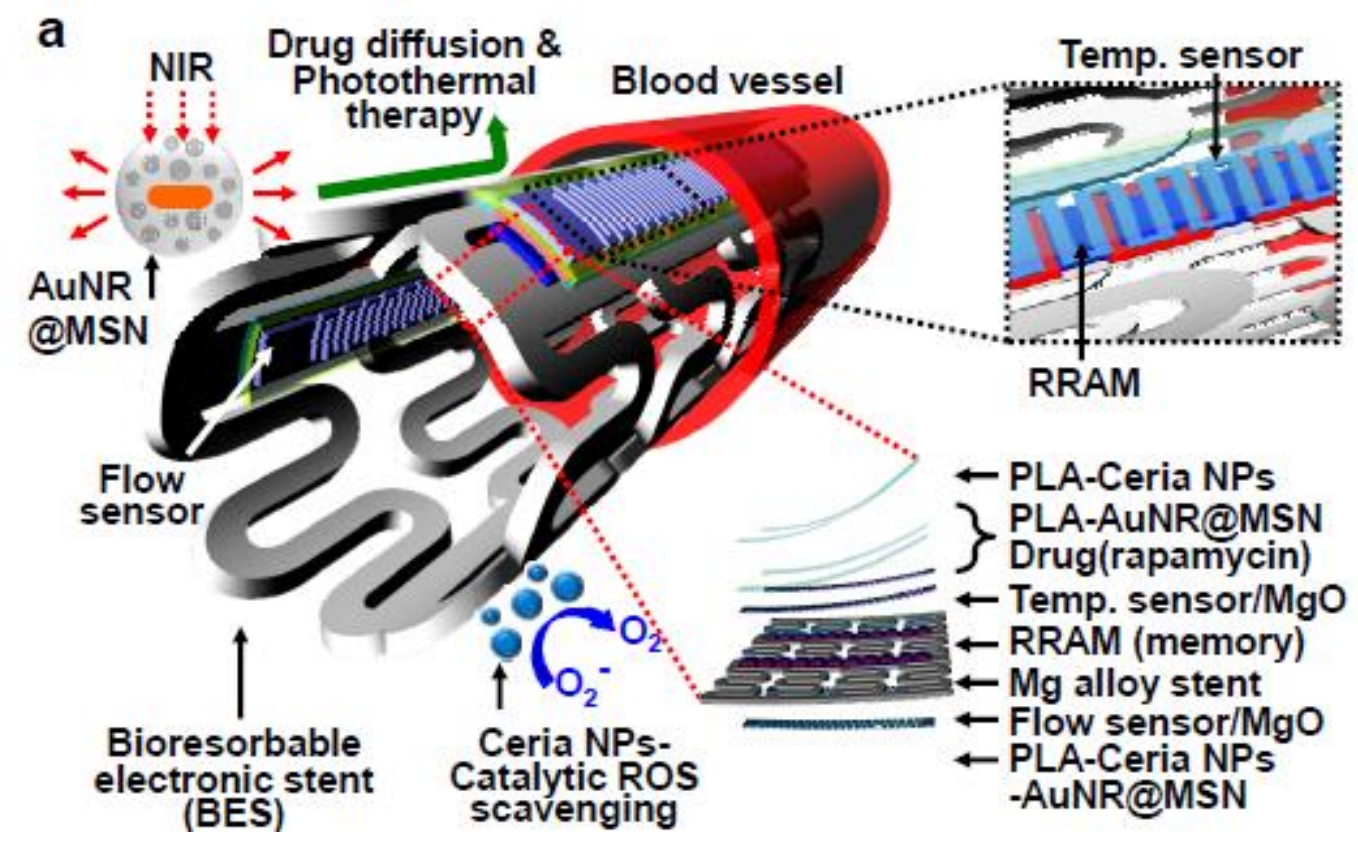

Fig. 6: Bioresorbable electronic stent [87]. 
Drug free

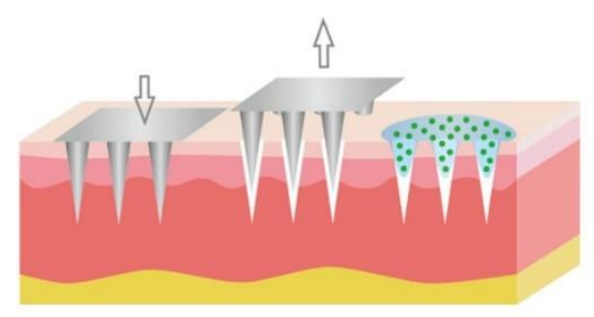

a

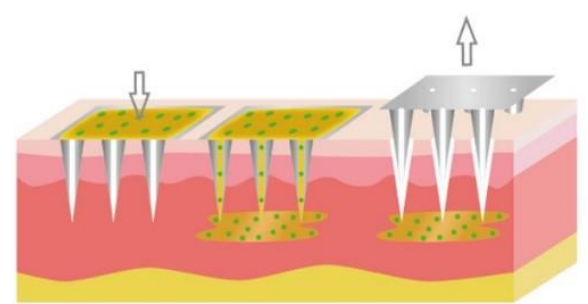

b

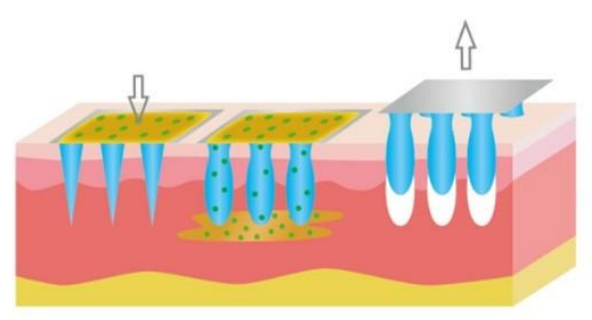

C

\section{Drug loaded}
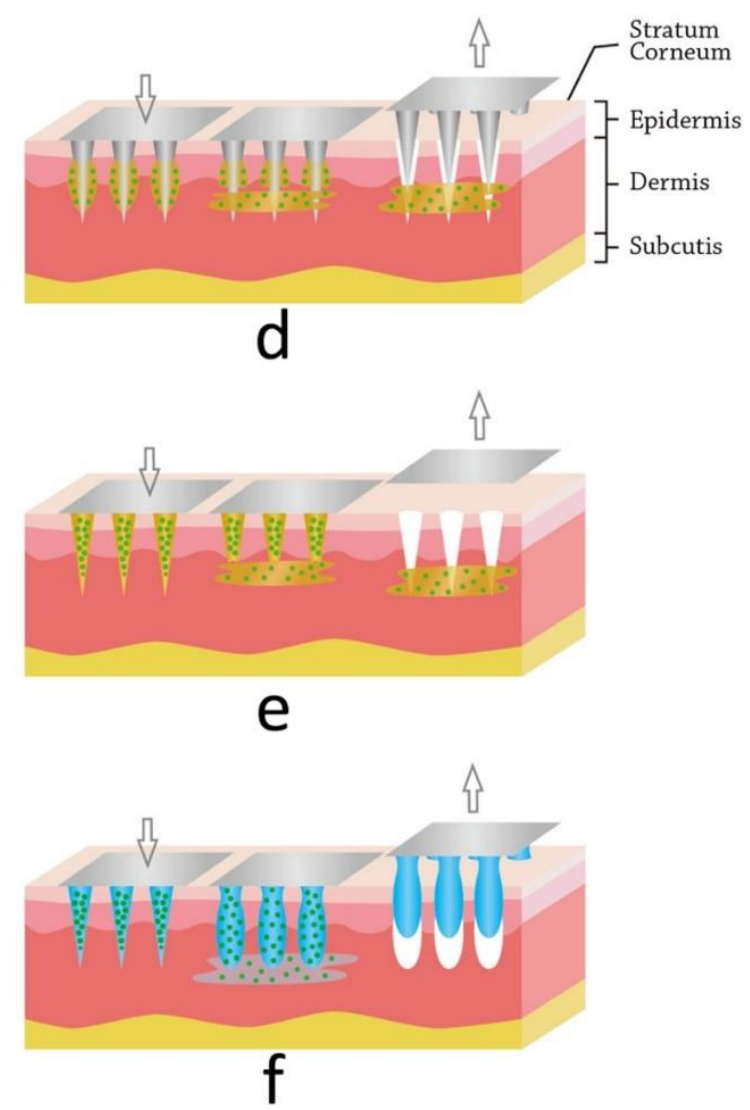

Fig. 7: Different types of MNs array used in transdermal drug delivery. a) Solid, b) hollow, c) reservoir-based swelling, d) drug loaded - including coated, e) dissolvable, f) drug encapsulated swelling. The working principle between reservoir-based swelling and encapsulated drug swelling is the same, the main difference stands in the reservoir position: in the first, the reservoir adheres to the backing of the MNs patch, in the latter the drug is encapsulated within the needle body [110]. 
A

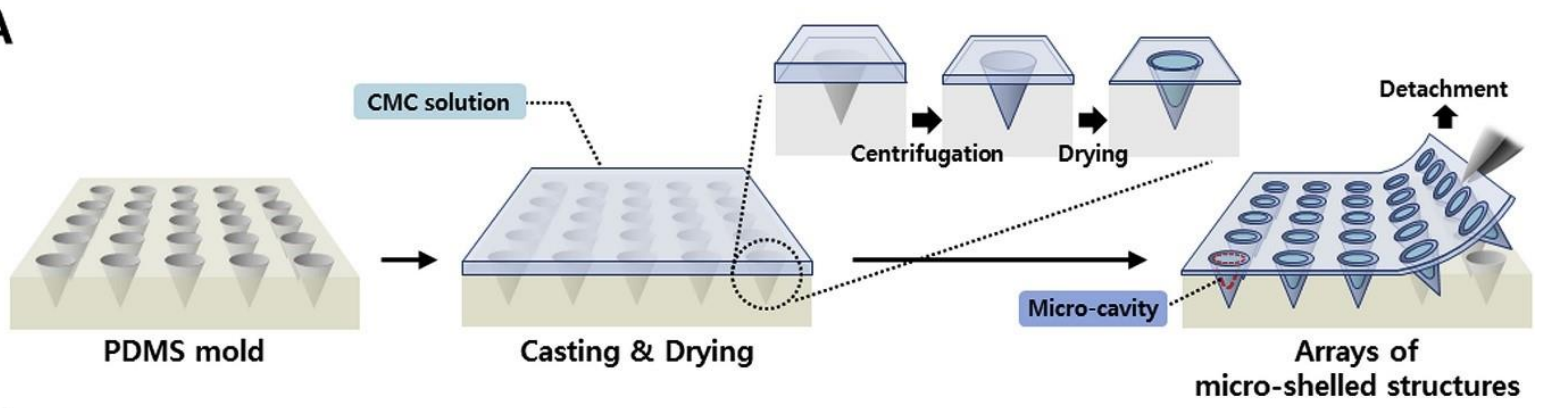

B

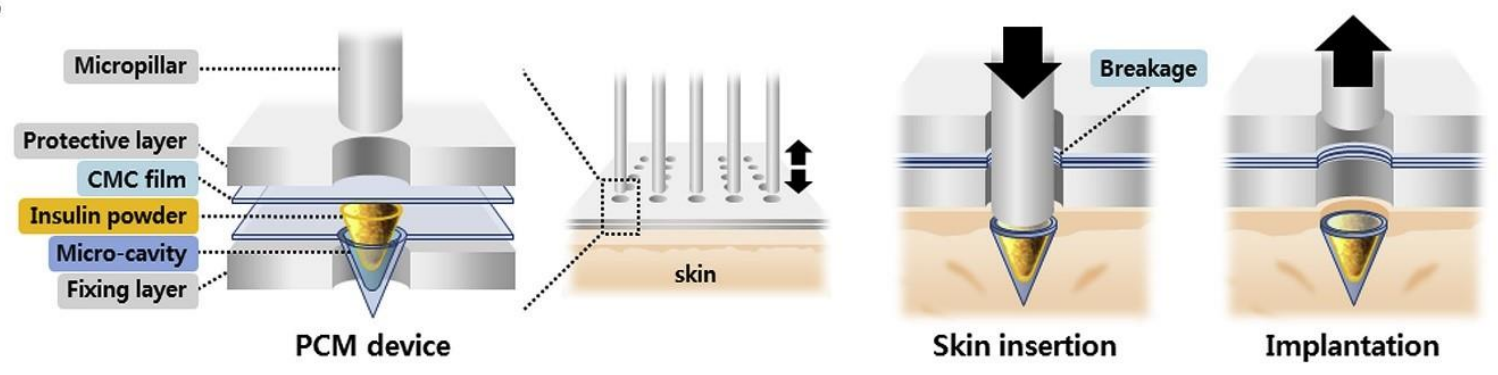

C

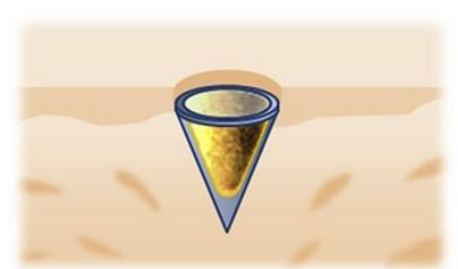

Implanted PCM

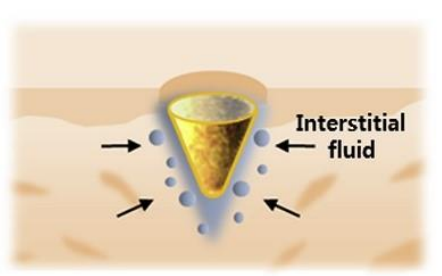

Dissolution of the micro-shell structure

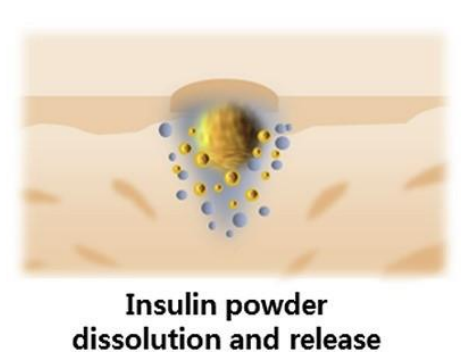

Fig. 8: PCMs system developed by Kim et al. a) Needles preparation, b) insulin powder loading and device administration c) mechanism for insulin delivery [114]. 
(a)

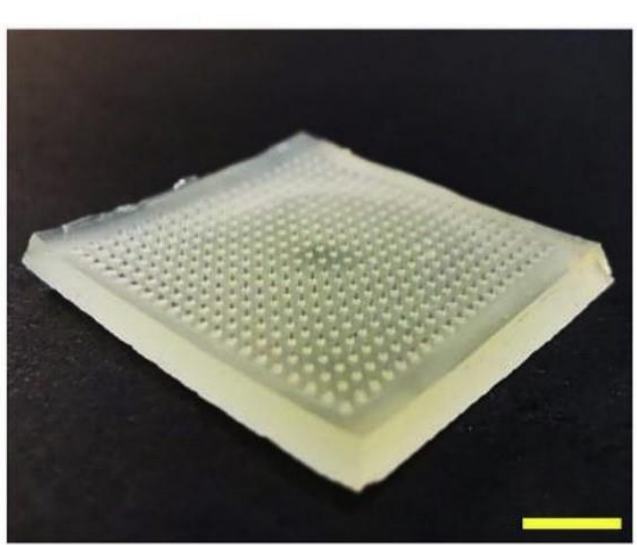

(c)

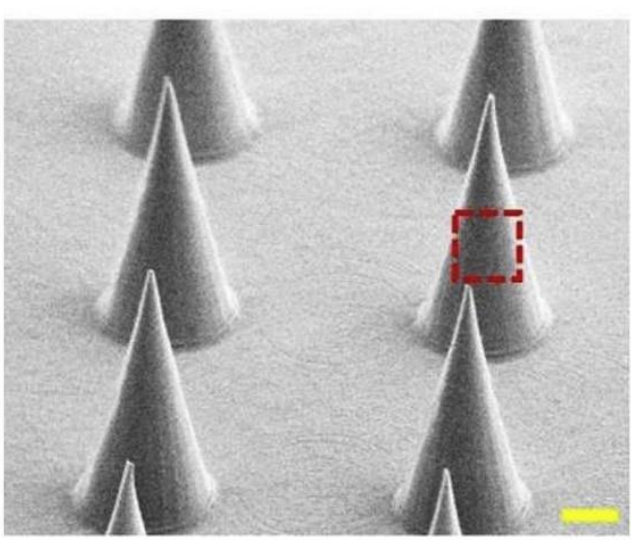

(b)

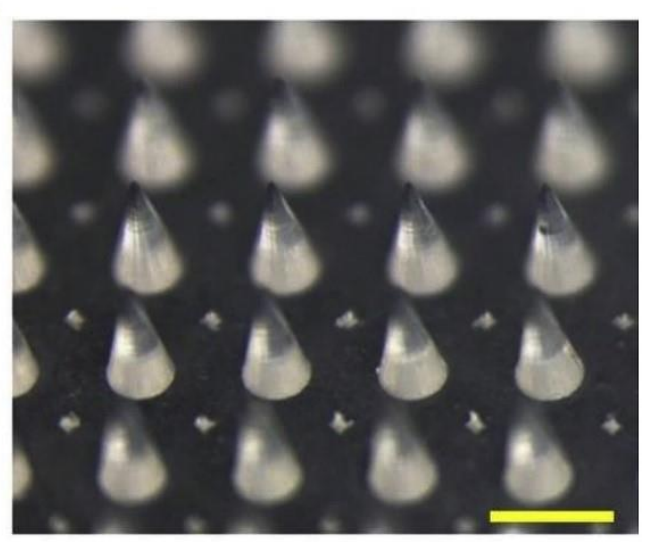

(d)

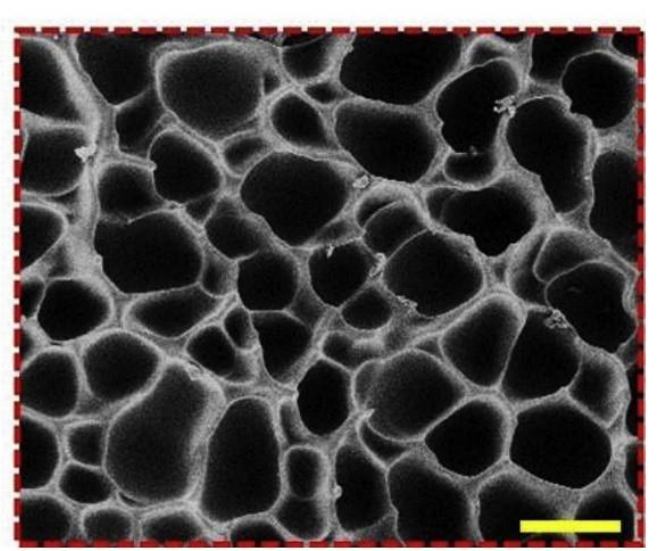

Fig. 9: Chitosan microneedles by Chi et al. a) Optical image, b) micrograph, c) SEM and d) surface microstructure[153]. 